\title{
Arrangement of hyperplanes I: Rational functions and Jeffrey-Kirwan residue
}

\author{
Michel Brion and Michèle Vergne
}

\section{Introduction}

Consider the space $R_{\Delta}$ of rational functions of $r$ variables with poles on an arrangement of hyperplanes $\Delta$. It is important to study the decomposition of the space $R_{\Delta}$ under the action of the ring of differential operators with constant coefficients. In the one variable case, a rational function of $z$ with poles at most on $z=0$ is written uniquely as $\phi(z)=\operatorname{Princ}(\phi)(z)+\psi(z)$ where $\operatorname{Princ}(\phi)(z)=\sum_{n<0} a_{n} z^{n}$ is the principal part of $\phi(z)$ and $\psi(z)=\sum_{n \geq 0} a_{n} z^{n}$ is the polynomial part of $\phi(z)$. Remark that the space

$$
G=\left\{\phi(z)=\sum_{n<0} a_{n} z^{n}\right\}
$$

of principal parts is free under the action of $\partial / \partial z$ while the space of polynomials is evidently a torsion module. Furthermore, the function $1 / z$ is the unique function which cannot be written as a derivative.

We show similarly, in the case of several variables, that there is a well determined decomposition of $R_{\Delta}$ as

$$
R_{\Delta}=G_{\Delta} \oplus N G_{\Delta}
$$

where $G_{\Delta}$ is a free module under the action of the ring of differential operators with constant coefficients, and $N G_{\Delta}$ is the torsion submodule. Here the space $G_{\Delta}$ can be characterized as the space of rational functions with a zero at infinity in all directions. Let us describe more precisely the space $G_{\Delta}$. We need more notations.

Let $V$ be a finite dimensional vector space over a field $k$, of characteristic zero. Let $r=\operatorname{dim} V$. Let $\Delta$ be a finite subset of nonzero elements of $V$. 
Consider the union of hyperplanes in $V^{*}$ :

$$
\mathcal{H}^{*}(\Delta):=\bigcup_{\alpha \in \Delta}\left\{z \in V^{*},\langle z, \alpha\rangle=0\right\}
$$

and the ring $R_{\Delta}$ of rational functions on $V^{*}$ with poles contained in $\mathcal{H}^{*}(\Delta)$. We denote by $G_{\Delta}$ the subspace of $R_{\Delta}$ spanned by the elements

$$
\frac{1}{\prod_{\alpha \in \kappa} \alpha^{n_{\alpha}}}
$$

where $\kappa$ is a subset of $\Delta$ generating $V$ and where the $n_{\alpha}$ are positive integers. It turns out that $G_{\Delta}$ is the subspace of $R_{\Delta}$ consisting of functions that vanish at infinity in any direction. It is a graded vector space with highest graded part $G_{\Delta}[-r]:=S_{\Delta}$. Furthermore, $S_{\Delta}$ is the linear span of the

$$
\phi_{\sigma}=\frac{1}{\prod_{\alpha \in \sigma} \alpha}
$$

where $\sigma$ ranges over all bases of $\Delta$.

As the space $G_{\Delta}$ is a direct factor in $R_{\Delta}$, under the action of the ring $S\left(V^{*}\right)$ of differential operators with constant coefficients, there is a natural projection Res $e_{\Delta}$ from $R_{\Delta}$ to $S_{\Delta}$ that we call the Jeffrey-Kirwan residue. The name Residue is justified by the fact that the kernel of the map Res $\Delta$ is the space of derivatives, and by a generalization of the Cauchy formula. Any $S\left(V^{*}\right)$-morphism from $G_{\Delta}$ to another $S\left(V^{*}\right)$-module is entirely determined by its value on $S_{\Delta}$, and this morphism exists provided certain linear relations between the $\phi_{\sigma}$ are satisfied. The space $S_{\Delta}$ is isomorphic to the top degree component of the "Orlik-Solomon algebra" associated to the hyperplane arrangement $\mathcal{H}^{*}(\Delta)$; as a consequence, we produce bases of $S_{\Delta}$ consisting of certain $\phi_{\sigma}$. Their dual bases can be described in terms of iterated residues, as shown by Szenes (see [7] and section 4).

If $k=\mathbb{R}$, then $G_{\Delta}$ occurs as the space of Laplace transforms of locally polynomial functions with possible discontinuities on hyperplanes generated by $r-1$ elements of $\Delta$. The Laplace transform intertwines the action of $S\left(V^{*}\right)$ on locally polynomial functions by multiplication, with its action on $G_{\Delta}$ by differential operators with constant coefficients. We study the jumps of locally polynomial functions in terms of the poles of their Laplace transforms. As a consequence, we show that a locally polynomial function is continuous if and only if its Laplace transform vanishes at order 2 in any direction. We 
also construct inverses of the Laplace transform, using our description of $G_{\Delta}$ by generators and relations.

Many of the statements proved in this article are already implicitly stated in Jeffrey-Kirwan articles [4] and [5]. However, we felt the need, for applications, to clarify some of their statements. The main application will be an algebraic construction of Eisenstein series: to each rational function with poles on hyperplanes, we will associate a periodic meromorphic function in several variables. This will be treated in part II of this article.

Applications to the Poisson formula will be given in another article.

Our interest in the space of functions $R_{\Delta}$ and their Laplace transforms comes from the study of integrals over symplectic spaces of equivariant cohomology classes. Let $(M, \Omega)$ be a compact symplectic manifold, with an Hamiltonian action of a torus $T$. Let $f: M \rightarrow \mathfrak{t}^{*}$ be the moment map. Let $X \in \mathfrak{t}$. Let $\Omega(X)=\langle f, X\rangle+\Omega$ be the equivariant symplectic form. Let $\alpha(X)$ be an equivariant closed form on $M$. Consider the integral

$$
I(X)=\int_{M} \alpha(X) e^{\Omega(X)} .
$$

Assume for simplicity that the set $F$ of fixed points for the action of $T$ on $M$ is finite. For $p \in F$, let $\Delta_{p} \subset \mathfrak{t}^{*}$ be the set of weights for the action of $T$ in the tangent space $T_{p} M$. Then, by the localisation formula in equivariant cohomology, we have

$$
I(X)=\sum_{p \in F} \phi_{p}(X) e^{\langle f(p), X\rangle}
$$

where each $\phi_{p}$ is in the ring $R_{\Delta_{p}}$.

If $\xi$ is a regular value of $f$, we can consider the reduced space $M_{\text {red }}(\xi)=$ $f^{-1}(\xi) / T$ with reduced symplectic structure $\Omega_{\xi}$. The equivariant cohomology class of $\alpha(X)$ gives rise to a de Rham cohomology class $\alpha_{\xi}$ on $M_{\text {red }}(\xi)$. Consider the function

$$
r(\xi)=\int_{M_{r e d}(\xi)} \alpha_{\xi} e^{\Omega_{\xi}}
$$

This function is defined for regular values of $\xi$. It is important to determine this function and its jumps when crossing walls of singular values of the moment map. The functions $I(X)$ and $r(\xi)$ are related by the Laplace transform. Thus it is important to study jumps of Laplace transforms of functions in the space $G_{\Delta}$.

We thank Michel Duflo for his comments on this article, and Bernard Malgrange for his decisive help in the proof of Theorem 1. 


\section{Rational functions with poles on hyperplanes: Jeffrey-Kirwan residue}

Let $V$ be a finite dimensional vector space over a field $k$, of characteristic zero. Let $r=\operatorname{dim} V$. We denote by $S(V)$ the symmetric algebra of $V$. Let $V^{*}$ be the dual space. We identify $S(V)$ with the ring of polynomial functions on $V^{*}$. Let $\Delta \subset V$ be a finite subset of nonzero elements, which spans $V$. We denote by

$$
R_{\Delta}:=\Delta^{-1} S(V)
$$

the ring generated over $S(V)$ by inverting the linear functions $\alpha \in \Delta$. This is a ring graded by the degree (positive or negative). Consider the union of hyperplanes in $V^{*}$ :

$$
\mathcal{H}^{*}(\Delta):=\bigcup_{\alpha \in \Delta}\left\{z \in V^{*},\langle\alpha, z\rangle=0\right\}
$$

and the open subset

$$
V_{\text {reg, }, \Delta}^{*}=V^{*}-\mathcal{H}^{*}(\Delta)
$$

of $(\Delta)$-regular elements in $V^{*}$. Then $R_{\Delta}$ is the ring of rational functions on $V^{*}$ with poles contained in the union of hyperplanes $\mathcal{H}^{*}(\Delta)$. Functions in $R_{\Delta}$ are defined on the set $V_{\text {reg }, \Delta}^{*}$ of regular elements.

Let $\mathcal{D}$ be the ring of differential operators on $V^{*}$ with polynomial coefficients. Recall that the ring $\mathcal{D}$ is generated by its subrings $S(V)$ of polynomial functions on $V^{*}$, and $S\left(V^{*}\right)$ of differential operators on $V^{*}$ with constant coefficients. Observe that $S(V)$ and $R_{\Delta}$ are graded $\mathcal{D}$-modules.

If $\phi \in R_{\Delta}$ and if $y \in V^{*}$ is a regular element, then $t \mapsto \phi(y+t z)$ is a rational function for any $z \in V^{*}$. We say that $\phi$ vanishes at infinity if the rational function $t \mapsto \phi(y+t z)$ is 0 at $\infty$ for all regular $y \in V^{*}$ and for all $z \in V^{*}$.

Let $\kappa$ be a subset of $\Delta$. The subset $\kappa$ is called generating if the $\alpha \in \kappa$ generate the vector space $V$. It is called a basis of $\Delta$, if the $\alpha \in \kappa$ form a basis of $V$. We denote by $\mathcal{B}(\Delta)$ the set of bases of $\Delta$.

For $\kappa \subset \Delta$, set

$$
\phi_{\kappa}:=\frac{1}{\prod_{\alpha \in \kappa} \alpha} .
$$

We denote by $G_{\Delta}$ the subspace of $R_{\Delta}$ spanned by the

$$
\frac{1}{\prod_{\alpha \in \kappa} \alpha^{n_{\alpha}}}
$$


where $\kappa$ is generating and the $n_{\alpha}$ are positive integers. Then $G_{\Delta}$ is a graded vector space with highest graded part $S_{\Delta}$ (in degree $-r$ ). Furthermore, $S_{\Delta}$ is the linear span of the $\phi_{\sigma}$ where $\sigma$ ranges over all bases of $\Delta$.

Clearly, any function in the space $G_{\Delta}$ vanishes at infinity. We will prove that the converse holds in Theorem 1 below.

Remark. The space $G_{\Delta}$ is contained in $\sum_{j \geq r} R_{\Delta}[-j]$ but is strictly smaller if $r>1$. For example, if $\alpha \in \Delta$, then $\alpha^{-r}$ is never in $G_{\Delta}$.

We denote by $N G_{\Delta}$ the subspace of $R_{\Delta}$ spanned by the

$$
\frac{\psi}{\prod_{\alpha \in \kappa} \alpha^{n_{\alpha}}}
$$

where $\psi \in S(V), \kappa$ is not generating and the $n_{\alpha}$ are non-negative integers.

Remark that the subspace $N G_{\Delta}$ of $R_{\Delta}$ is stable under the action of $\mathcal{D}$, whereas $G_{\Delta}$ is stable under the action of $S\left(V^{*}\right)$ by differential operators with constant coefficients.

Theorem 1 We have a direct sum decomposition of $S\left(V^{*}\right)$-modules

$$
R_{\Delta}=G_{\Delta} \oplus N G_{\Delta} .
$$

Moreover, the space $G_{\Delta}$ is a free $S\left(V^{*}\right)$-module, and is freely generated by $S_{\Delta}$, while the space $N G_{\Delta}$ is the torsion submodule. Finally, $G_{\Delta}$ is the space of functions in $R_{\Delta}$ which vanish at infinity.

For this we prove a succession of lemmas.

Lemma 2 The $S\left(V^{*}\right)$-module $G_{\Delta}$ is generated by $S_{\Delta}$. Moreover, we have $R_{\Delta}=G_{\Delta}+N G_{\Delta}$.

Proof. Observe that the $S\left(V^{*}\right)$-module generated by $S_{\Delta}$ is the span of the elements

$$
\frac{1}{\prod_{\beta \in \sigma} \beta^{n_{\beta}}}
$$

where $\sigma \in \mathcal{B}(\Delta)$ and where each $n_{\beta}$ is a positive integer. To prove the first assertion, it is enough to check that this vector space is stable by multiplication by $1 / \alpha^{n}$ where $\alpha \in \Delta$. For this, write $\alpha=\sum_{\beta \in \sigma} c_{\alpha \beta} \beta$. Then we have

$$
\frac{1}{\alpha^{n} \prod_{\beta \in \sigma} \beta^{n_{\beta}}}=\sum_{\beta \in \sigma} \frac{c_{\alpha \beta}}{\alpha^{n+1} \beta^{n_{\beta}-1} \prod_{\gamma \in \sigma, \gamma \neq \beta} \gamma^{n_{\gamma}}} .
$$


If $\beta \in \sigma$ is such that $n_{\beta}=1$, then the corresponding term in the right-hand side is in the $S\left(V^{*}\right)$-module generated by $G_{\Delta}[-r)$ : indeed, if $c_{\alpha \beta} \neq 0$ then $\sigma \cup\{\alpha\} \backslash\{\beta\}$ is a basis of $\Delta$. On the other hand, if $n_{\beta}>1$ then our term is the inverse of $\alpha^{n+1} \prod_{\beta \in \sigma} \beta^{n_{\beta}^{\prime}}$ with $n_{\beta}^{\prime} \geq 1$ and $\sum_{\beta \in \sigma} n_{\beta}^{\prime}=\left(\sum_{\beta \in \sigma} n_{\beta}\right)-1$. So the assertion follows by induction on $\sum_{\beta \in \sigma} n_{\beta}$.

Similarly, any element of $R_{\Delta}=\Delta^{-1} S(V)$ is a linear combination of elements

$$
\phi=\frac{\psi}{\prod_{\alpha \in \kappa} \alpha^{n_{\alpha}}}
$$

where $\psi \in S(V), \kappa$ is linearly independent and the $n_{\alpha}$ are positive integers. If moreover $\kappa$ is not generating, then $\phi$ is in $N G_{\Delta}$. If $\kappa$ is generating, then we can express $\psi$ as a polynomial in the variables $\alpha \in \kappa$, and we obtain $\phi \in G_{\Delta}+N G_{\Delta}$.

Lemma 3 The $S\left(V^{*}\right)$-module $R_{\Delta} / N G_{\Delta}$ is free.

Proof. Observe that $R_{\Delta} / N G_{\Delta}$ is a $\mathcal{D}$-module. Furthermore, it is spanned (as a vector space) by the images of

$$
\frac{1}{\prod_{\alpha \in \sigma} \alpha^{n_{\alpha}}}
$$

where $\sigma$ is a basis of $\Delta$, and the $n_{\alpha}$ are positive integers. It follows that the $\mathcal{D}$-module $R_{\Delta} / N G_{\Delta}$ is generated by the images $\overline{\phi_{\sigma}}$ of the $\phi_{\sigma}(\sigma \in \mathcal{B}(\Delta))$. Observe that $\overline{\phi_{\sigma}}$ is killed by $V$; thus, the $\mathcal{D}$-module $\mathcal{D} \overline{\phi_{\sigma}}$ is a non zero quotient of $\mathcal{D} / \mathcal{D} V$. The latter is a simple $\mathcal{D}$-module, isomorphic to $S\left(V^{*}\right)$; therefore, $\mathcal{D} \overline{\phi_{\sigma}}$ is isomorphic to $S\left(V^{*}\right)$, too. Iterating this argument, we construct an ascending filtration of the $\mathcal{D}$-module $R_{\Delta} / N G_{\Delta}$, each submodule being generated by certain $\overline{\phi_{\sigma}}$ 's, with successive quotients isomorphic to $S\left(V^{*}\right)$.

Lemma 4 The subspace $S_{\Delta}$ intersects $N G_{\Delta}$ trivially.

Proof. We argue by induction on the number of elements in $\Delta$. We may assume that $\Delta$ contains no proportional elements. Let $\phi \in S_{\Delta}$. Write

$$
\phi=\sum_{\sigma \in \mathcal{B}(\Delta)} \frac{c_{\sigma}}{\prod_{\alpha \in \sigma} \alpha}
$$


and consider $\phi$ as a rational function on $V^{*}$. Observe that the poles of $\phi$ are simple and along the hyperplanes $\alpha=0(\alpha \in \Delta)$. Choose $\alpha$ among the poles of $\phi$. Choose a decomposition $V=k \alpha \oplus V_{0}$. Then $Q(V)$ (the fraction field of $S(V)$ ) is identified with the field of rational functions in the variable $\alpha$, with coefficients in $Q\left(V_{0}\right)$. Therefore, we have a restriction map $S(V) \rightarrow$ $S\left(V_{0}\right): \phi \mapsto \phi_{0}$. Consider the image $\Delta_{0}$ of $\Delta \backslash\{\alpha\}$ in $V_{0}$. The restriction map extends to an homomorphism $(\Delta \backslash\{\alpha\})^{-1} S(V) \rightarrow \Delta_{0}^{-1} S\left(V_{0}\right)$ by restriction to generic points. We have also a residue map $\operatorname{Res}_{\alpha}: Q(V) \rightarrow Q\left(V_{0}\right)$ with respect to the variable $\alpha$, defined by the formula

$$
\operatorname{Res}_{\alpha}(\phi)=\frac{1}{(K-1) !}\left(\left(\frac{\partial}{\partial \alpha}\right)^{K-1}\left(\alpha^{K} \phi\right)\right)_{0}
$$

for any integer $K$ such that $\alpha^{K} \phi \in R_{\Delta \backslash\{\alpha\}}$.

As $\alpha$ is a simple pole of $\phi$, we have simply

$$
\operatorname{Res}_{\alpha}(\phi)=\sum_{\sigma, \alpha \in \sigma} \frac{c_{\sigma}}{\prod_{\beta \in \sigma, \beta \neq \alpha} \beta_{0}}
$$

where $\beta_{0}$ denotes the image of $\beta$ in $V_{0}$. If $\sigma$ is a basis of $\Delta$ which contains $\alpha$, then $(\sigma \backslash\{\alpha\})_{0}$ is a basis of $\Delta_{0}$. Therefore, $\operatorname{Res}_{\alpha}(\phi)$ is in $G_{\Delta_{0}}$.

Consider a generator

$$
u=\frac{\psi}{\prod_{\beta \in \kappa} \beta^{n_{\beta}}}
$$

of $N G_{\Delta}$, with $\psi \in S(V)$ and $\kappa$ non generating. Write

$$
u=\frac{\psi}{\alpha^{K} \prod_{\beta \in \kappa, \beta \neq \alpha} \beta^{n_{\beta}}} .
$$

If $K=0$, then $\operatorname{Res}_{\alpha}(u)=0$. If $K>0$, the set $\kappa$ contains $\alpha$ and is non generating. Thus, its restriction $\kappa_{0}$ is non generating. We see that $\operatorname{Res}_{\alpha}(u)$ can be written as

$$
\operatorname{Res}_{\alpha}(u)=\frac{\psi^{\prime}}{\prod_{\beta_{0} \in \kappa_{0}} \beta_{0}^{n_{\beta}+K-1}} .
$$

for some $\psi^{\prime} \in S\left(V_{0}\right)$, so that $\operatorname{Res}_{\alpha}(u) \in N G_{\Delta_{0}}$.

If $\phi \in G_{\Delta} \cap N G_{\Delta}$, it follows from the above discussion that $\operatorname{Res}_{\alpha}(\phi) \in$ $G_{\Delta_{0}} \cap N G_{\Delta_{0}}$. Therefore, by the induction hypothesis, we have $\operatorname{Res}_{\alpha}(\phi)=0$ : thus, $\phi$ has no pole along $\alpha=0$. By the beginning of the proof, $\phi$ has no pole at all, so that $\phi=0$. 
Lemma 5 If $\phi \in R_{\Delta}$ vanishes at infinity, then $\phi$ is in $G_{\Delta}$.

Proof. First we claim that the space of functions which vanish at infinity is stable by the action of $S\left(V^{*}\right)$. Indeed, let $\phi \in R_{\Delta}$ vanish at infinity. Write

$$
\phi=\frac{\psi}{\prod_{\alpha \in \Delta} \alpha^{n_{\alpha}}}
$$

where $\psi \in S(V)$. For $z \in V^{*}$, set

$$
n(z):=\sum_{\alpha,\langle\alpha, z\rangle \neq 0} n_{\alpha} .
$$

The assumption that $\phi$ vanishes at infinity means that

$$
\operatorname{deg}(t \mapsto \psi(y+t z))<n(z)
$$

for all regular $y$ and for all $z$ in $V^{*}$. Let $w \in V^{*}$; then, for all $u \in k$ such that $y+u w$ is regular, we also have

$$
\operatorname{deg}(t \mapsto \psi(y+t z+u w))<n(z)
$$

and therefore, the function

$$
\frac{\partial(w) \psi}{\prod_{\alpha \in \Delta} \alpha^{n_{\alpha}}}
$$

vanishes at infinity. Now

$$
\partial(w) \phi=\frac{\partial(w) \psi}{\prod_{\alpha \in \Delta} \alpha^{n_{\alpha}}}-\sum_{\alpha \in \kappa} \frac{n_{\alpha}\langle\alpha, w\rangle}{\Delta} \phi
$$

which implies our claim.

Assume now that there exists a non-zero $\phi \in N G_{\Delta}$ which vanishes at infinity. As in the proof of Lemma 2, we can write

$$
\phi=\sum_{\kappa} \phi_{\kappa}
$$

where the sum is over all linearly independent subsets $\kappa \subset \Delta$ which are not bases, and where each $\phi_{\kappa}$ is in $\kappa^{-1} S(V)$. Furthermore, we may assume that the number of $\kappa$ such that $\phi_{\kappa}$ is non-zero is minimal (among all possible decompositions of all non-zero $\phi \in N G_{\Delta}$ which vanish at infinity). 
Choose $\kappa_{0}$ such that $\phi_{\kappa_{0}} \neq 0$, and choose a non-zero $z_{0} \in V^{*}$ such that $\left\langle\alpha, z_{0}\right\rangle=0$ for all $\alpha \in \kappa_{0}$. Then $\partial^{n}\left(z_{0}\right) \phi_{\kappa_{0}}=0$ for large $n$. But all successive derivatives of $\phi$ vanish at infinity and are in $N G_{\Delta}$. Moreover,

$$
\partial^{n}\left(z_{0}\right) \phi=\sum_{\kappa \neq \kappa_{0}} \partial^{n}\left(z_{0}\right) \phi_{\kappa}
$$

is a decomposition with fewer terms than $\phi$. Thus, $\partial^{n}\left(z_{0}\right) \phi=0$ for some positive $n$.

Choose $n$ minimal with this property, and set $\psi:=\partial^{n-1}\left(z_{0}\right) \phi$. Then $\psi$ is a non-zero element of $N G_{\Delta}$ which vanishes at infinity, and $\partial\left(z_{0}\right) \psi=0$. But then the function $t \mapsto \psi\left(y+t z_{0}\right)$ is constant for any $y \in V^{*}$, a contradiction.

The space $S_{\Delta}$ is generated by the elements $\phi_{\sigma}$ where $\sigma$ ranges over $\mathcal{B}(\Delta)$. However, there are linear relations among the elements $\phi_{\sigma}$. Indeed, let $\sigma$ be a basis of $\Delta$. If $\alpha \in \Delta \backslash \sigma$ and

$$
\alpha=\sum_{\beta \in \sigma} c_{\alpha \beta} \beta
$$

is the expansion of $\alpha$ in the basis $\sigma$, then $\sigma \cup\{\alpha\} \backslash\{\beta\}$ is a basis if and only if $c_{\alpha \beta}$ is non zero, and we have

$$
\phi_{\sigma}=\sum_{\beta \in \sigma, c_{\alpha \beta} \neq 0} c_{\alpha \beta} \phi_{\sigma \cup\{\alpha\} \backslash\{\beta\}} .
$$

In section 4 , we will prove that the linear relations between the elements $\phi_{\sigma}$ are generated by the relations above.

We can now define the Jeffrey-Kirwan residue map: denote by

$$
\hat{R}_{\Delta}:=\Delta^{-1} \hat{S}(V)
$$

the ring generated over the ring $\hat{S}(V)$ of formal power series, by inverting the linear functions $\alpha \in \Delta$. Define the Taylor expansion at order $K$ as the projection

$$
\text { Taylor }_{[\leq K]}: \hat{R}_{\Delta} \rightarrow \bigoplus_{j \leq K} R_{\Delta}[j]
$$

Using Taylor $_{[\leq-r]}$, we project the space $\hat{R}_{\Delta}$ to $R_{\Delta}[\leq-r]$. Then using the direct sum decomposition

$$
R_{\Delta}=G_{\Delta} \oplus N G_{\Delta}
$$


we obtain a projection map

$$
\operatorname{Princ}_{\Delta}: \hat{R}_{\Delta} \rightarrow G_{\Delta}
$$

by composing both projections $\hat{R}_{\Delta} \rightarrow R_{\Delta}[\leq-r] \rightarrow G_{\Delta}$.

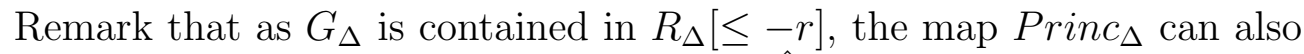
be defined as the composition of Taylor $_{[\leq K]}: \hat{R}_{\Delta} \rightarrow R_{\Delta}[\leq K]$ for any index $K \geq-r$, followed by the projection $R_{\Delta}[\leq-K] \rightarrow G_{\Delta}$.

Definition 6 The Jeffrey-Kirwan residue map

$$
\operatorname{Res}_{\Delta}: \hat{R}_{\Delta} \rightarrow S_{\Delta}
$$

is defined to be the composite of the projection Princ $\Delta$ followed by the projection of $G_{\Delta}$ on $S_{\Delta}$.

In other words, the map Res $s_{\Delta}$ is the identity on $S_{\Delta}$ and vanishes on $\oplus_{j \neq-r} R_{\Delta}[j]$ and on $N G_{\Delta}$ as well. We can determine easily the map $R_{e} s_{\Delta}$ on $\hat{R}_{\Delta}$ by first projecting on $R_{\Delta}[-r]$, then, using the fact that $R e s_{\Delta}$ vanishes on $N G_{\Delta}$, projecting further on $G_{\Delta}[-r]=S_{\Delta}$.

Consider the subspace $V^{*} R_{\Delta}$ spanned by derivatives of elements of $R_{\Delta}$; it is a submodule of $R_{\Delta}$ under the action of $S\left(V^{*}\right)$.

Proposition 7 We have

$$
V^{*} R_{\Delta}=N G_{\Delta} \oplus \bigoplus_{j<-r} G_{\Delta}[j]
$$

In particular, we have

$$
R_{\Delta}=V^{*} R_{\Delta} \oplus S_{\Delta}, \hat{R}_{\Delta}=V^{*} \hat{R}_{\Delta} \oplus S_{\Delta}
$$

and the kernel of Res $s_{\Delta}$ is $V^{*} \hat{R}_{\Delta}$.

Proof. From Theorem [ ] we obtain

$$
V^{*} R_{\Delta}=V^{*} N G_{\Delta} \oplus V^{*} G_{\Delta}=V^{*} N G_{\Delta} \oplus \bigoplus_{j<-r} G_{\Delta}[j] .
$$

So it is sufficient to check that $N G_{\Delta}=V^{*} N G_{\Delta}$. For this, consider

$$
\phi=\frac{\psi}{\prod_{\alpha \in \kappa} \alpha^{n_{\alpha}}}
$$


where $\psi \in S(V)$ and where $\kappa$ is linearly dependent. Choose $y \in V^{*}$ such that $\langle y, \alpha\rangle=0$ for all $\alpha \in \kappa$. We can find $\Psi \in S(V)$ such that $\partial(y) \Psi=\psi$; then

$$
\phi=\partial(y)\left(\frac{\Psi}{\prod_{\alpha \in \kappa} \alpha^{n_{\alpha}}}\right) .
$$

In particular, the kernel of $\operatorname{Res}_{\Delta}$ is the space of derivatives. Using Res $s_{\Delta}$, we now obtain a multidimensional analogue of the Cauchy formula: for any meromorphic function $\phi$ of one variable $z$, and for any $y \neq 0$, we have

$$
(\operatorname{Princ} \phi)(y)=\operatorname{Res}_{z=0} \frac{\phi(z)}{y-z} \text {. }
$$

Let $y \in V^{*}$ be regular and let $\psi \in R_{\Delta}$. Set

$$
(C(y) \psi)(z):=\psi(y-z) .
$$

Then the rational function $C(y) \psi$ is defined at 0 , and thus its Taylor series at the origin is in $\hat{S}(V)$. To any $\phi \in \hat{R}_{\Delta}$, we associate the endomorphism $u(\phi)$ of $S_{\Delta}$ defined by

$$
(u(\phi) \psi)(z)=\operatorname{Res}_{\Delta}(\phi(z) \psi(y-z)) .
$$

Denoting by $m(\phi)$ the multiplication by $\phi$, then $u(\phi)$ is composition $\operatorname{Res}_{\Delta} \circ$ $m(\phi) \circ C(y)$. We consider its trace $\operatorname{Tr}_{S_{\Delta}}\left(\operatorname{Res}_{\Delta} \circ m(\phi) \circ C(y)\right)$.

Proposition 8 For any regular $y$ in $V^{*}$ and for any $\phi \in \hat{R}_{\Delta}$, we have

$$
\left(\operatorname{Princ}_{\Delta} \phi\right)(y)=\operatorname{Tr}_{S_{\Delta}}\left(\operatorname{Res}_{\Delta} \circ m(\phi) \circ C(y)\right) .
$$

Proof. First we consider the case where $\phi \in S_{\Delta}$. Then

$$
\operatorname{Res}_{\Delta}(\phi(z) \psi(y-z))=\phi(z) \psi(y)
$$

because $z \mapsto \psi(y-z)$ is defined at 0 . So $u(\phi)$ maps $\psi$ to $\psi(y) \phi$, and its trace is $\phi(y)=\operatorname{Princ}_{\Delta}(\phi)(y)$.

Now we assume that the formula holds for $\phi$, and we claim that it holds for $\partial(w) \phi$ where $w \in V^{*}$. Indeed, using the fact that $\operatorname{Res}_{\Delta}$ vanishes on derivatives, we obtain

$\operatorname{Res}_{\Delta}((\partial(w) \phi)(z) \psi(y-z))=-\operatorname{Res}_{\Delta}\left(\phi(z) \partial_{z}(w) \psi(y-z)\right)$ 


$$
=\operatorname{Res}_{\Delta}\left(\phi(z) \partial_{y}(w) \psi(y-z)\right)=\partial_{y}(w) \operatorname{Res}_{\Delta}(\phi(z) \psi(y-z))
$$

which implies the claim.

It follows that the formula holds for any $\phi \in G_{\Delta}$. If $\phi \in \widehat{N G_{\Delta}}$ then the left-hand side vanishes. On the other hand, the function $z \mapsto \psi(y-z)$ is in $\hat{S}(V)$; thus, $z \mapsto \phi(z) \psi(y-z)$ is in $\widehat{N G_{\Delta}}$ and the right-hand side vanishes, too.

Remark. More generally, let $A: \hat{R}_{\Delta} \rightarrow \hat{R}_{\Delta}$ be an operator which commutes with the action of $S\left(V^{*}\right)$. Then we have for any regular $y \in V^{*}$ and for any $\phi \in \hat{R}_{\Delta}$ :

$$
A\left(\operatorname{Princ}_{\Delta}(\phi)\right)(y)=\operatorname{Tr}_{S_{\Delta}}\left(\operatorname{Res}_{\Delta} \circ m(\phi) \circ C(y) \circ A\right)
$$

(the proof is the same).

Let us deduce from this (abstract) Cauchy formula, an explicit expression of $\operatorname{Princ}_{\Delta}(\phi)$ in terms of derivatives of elements of $S_{\Delta}$. For this, choose a basis $\left(\phi_{b}\right)_{b \in B}$ of $S_{\Delta}$ and denote by $\left(\phi^{b}\right)$ the dual basis. For $\phi \in \hat{R}_{\Delta}$ and $h \in V$, the function $y \mapsto e^{-\langle y, h\rangle} \phi(y)$ is in $\hat{R}_{\Delta}$. Moreover, the map

$$
h \mapsto\left\langle\phi^{b}, \operatorname{Res}_{\Delta}\left(e^{-h} \phi\right)\right\rangle:=D^{b}(\phi)(h)
$$

is easily seen to be polynomial. It thus defines a differential operator $D^{b}(\phi)$ on $V^{*}$.

Proposition 9 For any $\phi \in \hat{R}_{\Delta}$, and for any basis $\left(\phi_{b}\right)_{b \in B}$ of $S_{\Delta}$, we have

$$
\operatorname{Princ}_{\Delta}(\phi)=\sum_{b \in B} D^{b}(\phi) \cdot \phi_{b} .
$$

Proof. Let $y$ be a regular element of $V^{*}$. Then we have by the Cauchy formula:

$$
\begin{aligned}
& \operatorname{Princ}_{\Delta}(\phi)(y)=\operatorname{Tr}_{S_{\Delta}}\left(\operatorname{Res}_{\Delta} \circ m(\phi) \circ C(y)\right) \\
& =\sum_{b \in B}\left\langle\phi^{b}, \operatorname{Res}_{\Delta}\left(\phi(z) \phi_{b}(y-z)\right)\right\rangle .
\end{aligned}
$$

Now observe that $\phi_{b}(y-z)=\left(e^{-\partial(z)} \phi_{b}\right)(y)$. Thus, we have

$$
\left\langle\phi^{b}, \operatorname{Res}_{\Delta}\left(\phi(z) \phi_{b}(y-z)\right)\right\rangle=D^{b}(\phi) \cdot \phi_{b} .
$$


Remark that Propositions 13 and 14 below provide a basis $\left(\phi_{b}\right)_{b \in B}$ together with the dual basis $\left(\phi^{b}\right)_{b \in B}$. Thus we obtain an explicit expression of any element in $G_{\Delta}$ as a sum of successive derivatives of elements $\phi_{\sigma}$. This provides a way of separating variables.

Example. Let $V$ be a vector space with basis $\left(e_{1}, e_{2}\right)$. Let $\Delta$ be the ordered set

$$
\Delta=\left(e_{1}, e_{2}, e_{1}+e_{2}\right)
$$

The set $B$ of Proposition 13 according to this ordering consists of

$$
b_{1}=\left(e_{1}, e_{2}\right) \quad b_{2}=\left(e_{1}, e_{1}+e_{2}\right) .
$$

Furthermore, if $\sigma=\left\{e_{2}, e_{1}+e_{2}\right\}$, we have $\phi_{\sigma}=\phi_{b_{1}}-\phi_{b_{2}}$. Let

$$
\phi\left(z_{1}, z_{2}\right)=\frac{1}{z_{1} z_{2}\left(z_{1}+z_{2}\right)} .
$$

If $h=h_{1} e_{1}+h_{2} e_{2}$, the component of degree -2 of $e^{-h_{1} z_{1}-h_{2} z_{2}} \phi\left(z_{1}, z_{2}\right)$ is

$$
\frac{-h_{1} z_{1}-h_{2} z_{2}}{z_{1} z_{2}\left(z_{1}+z_{2}\right)}=-\frac{h_{1}}{z_{2}\left(z_{1}+z_{2}\right)}-\frac{h_{2}}{z_{1}\left(z_{1}+z_{2}\right)}=-h_{1} \phi_{b_{1}}+\left(h_{1}-h_{2}\right) \phi_{b_{2}} .
$$

We have indeed

$$
\frac{1}{z_{1} z_{2}\left(z_{1}+z_{2}\right)}=-\frac{\partial}{\partial z_{1}} \cdot \frac{1}{z_{1} z_{2}}+\left(\frac{\partial}{\partial z_{1}}-\frac{\partial}{\partial z_{2}}\right) \cdot \frac{1}{z_{1}\left(z_{1}+z_{2}\right)} .
$$

Remark. The residue that Jeffrey and Kirwan actually defined is a linear form over $S_{\Delta}$, defined in the case when $k=\mathbb{R}$. It depends on choices of chambers in $V$ and $V^{*}$. We will describe this residue in section 5 .

\section{Residue along a hyperplane}

Let us recall the notion of a residue map along a hyperplane.

Let $V_{0}$ be an hyperplane in $V$. We denote by $\Delta_{0}$ the subset $\Delta \cap V_{0}$. The space $V_{0}^{\perp}$ is a line in $V^{*}$. The fibers of the restriction map $V^{*} \rightarrow V_{0}^{*}$ are affine lines $z+V_{0}^{\perp}$. If $\phi$ is a rational function with poles on the set of hyperplanes $\Delta$, its restriction to the affine line $z+V_{0}^{\perp}$ is a rational function, except when the affine line $z+V_{0}^{\perp}$ is contained in the pole set of $\phi$ (in this case the restriction 
is nowhere defined). The residue at infinity of this rational function is well defined. More precisely, choose diffential forms of maximal degree $\omega$ on $V^{*}$, $\omega_{0}$ on $V_{0}^{*}$ and choose an equation $z_{0}$ of $V_{0}$, such that $\omega_{0}=\operatorname{int}\left(z_{0}\right) \omega$ where int is the contraction. Define the residue map

$$
\operatorname{Res}_{V / V_{0}}: \Delta^{-1} S(V) \otimes \wedge^{r} V \rightarrow \Delta_{0}^{-1} S\left(V_{0}\right) \otimes \wedge^{r-1} V_{0}
$$

by

$$
\operatorname{Res}_{V / V_{0}}(\phi \otimes \omega)(z)=-\operatorname{Res}_{t=\infty}\left(\phi\left(z+t z_{0}\right) d t\right) \otimes \omega_{0}
$$

for $z \in V^{*}$ (clearly, this only depends on the image of $z$ in $V^{*} / k z_{0}=V_{0}^{*}$ ). We now give a characterization of this map.

We identify $R_{\Delta_{0}}=\Delta_{0}^{-1} S\left(V_{0}\right)$ to a subalgebra of $R_{\Delta}$, so that $R_{\Delta}$ is a $R_{\Delta_{0}}$-module. We denote by $\Delta_{1}$ the complement of $\Delta_{0}$ in $\Delta$.

If $\nu=\left(\alpha_{j}, 1 \leq j \leq L\right)$ is a sequence of elements of $\Delta$ with possible repetitions, we set

$$
m_{\nu}:=\frac{1}{\prod_{j=1}^{L} \alpha_{j}} .
$$

We write $\nu \subset \Delta_{0}$ (resp. $\nu \subset \Delta_{1}$ ) if all elements $\alpha_{j}$ of the sequence $\nu$ are in $\Delta_{0}\left(\right.$ resp. $\left.\Delta_{1}\right)$.

Let $\omega \in \wedge^{r} V$. If $\beta \in \Delta_{1}$, there exists a unique $\omega_{0} \in \wedge^{r-1} V_{0}$ such that $\omega=\beta \wedge \omega_{0}$. We then write

$$
\omega_{0}=(d \beta)^{-1} \wedge \omega
$$

Proposition 10 The map $\operatorname{Res}_{V / V_{0}}$ is the unique $R_{\Delta_{0}}$-linear map

$$
r e s_{V / V_{0}}: R_{\Delta} \otimes \wedge^{r} V \rightarrow R_{\Delta_{0}} \otimes \wedge^{r-1} V_{0}
$$

such that, for $\omega \in \wedge^{r} V$,

1) for any $\beta \in \Delta_{1}$,

$$
\operatorname{res}_{V / V_{0}}\left(\frac{1}{\beta} \otimes \omega\right)=(d \beta)^{-1} \wedge \omega .
$$

2)

$$
\operatorname{res}_{V / V_{0}}(S(V) \otimes \omega)=0 .
$$

3) for any sequence $\nu \subset \Delta_{1}$ :

$$
r e s_{V / V_{0}}\left(m_{\nu} \otimes \omega\right)=0
$$

if the length of $\nu$ is strictly greater than 1 . 
Indeed, these properties are easily checked for the map $\operatorname{Res}_{V / V_{0}}$ defined above, and uniqueness follows from the following remark.

Proposition 11 We have

$$
R_{\Delta}=\Delta_{0}^{-1} S(V)+\sum_{\nu \subset \Delta_{1}} R_{\Delta_{0}} m_{\nu}
$$

Proof. Let $\psi \in S(V)$ and $\nu$ a sequence of elements of $\Delta$. Consider the element $\psi m_{\nu}$ of $R_{\Delta}=\Delta^{-1} S(V)$. If $\nu$ is contained in $\Delta_{0}$, or if $\psi \in S\left(V_{0}\right)$, we are already in the desired set. If $\alpha_{j} \in \nu$ is not in $\Delta_{0}$ and if $\psi$ is not in $S\left(V_{0}\right)$, then using the decomposition

$$
S(V)=S\left(V_{0}\right) \oplus \alpha_{j} S(V)
$$

we can strictly decrease the power of $\alpha_{j}$ in the expression of $m_{\nu}$.

We finally note some properties of the map $\operatorname{Res}_{V / V_{0}}$.

We extend the map Princ ${ }_{\Delta}: R_{\Delta} \rightarrow G_{\Delta}$ to a map

$$
\text { Princ }_{\Delta}: R_{\Delta} \otimes \wedge^{\max } V \rightarrow G_{\Delta} \otimes \wedge^{\max } V
$$

still denoted by $\operatorname{Princ}_{\Delta}$. In the same way we extend the map Res $s_{\Delta}$ to a map

$$
\operatorname{Res}_{\Delta}: R_{\Delta} \otimes \wedge^{\max } V \rightarrow S_{\Delta} \otimes \wedge^{\max } V .
$$

Proposition 12 The map Res $s_{V / V_{0}}$ is homogeneous of degree-1, and is compatible with the maps Princ and with the Jeffrey-Kirwan residue. More explicitly:

1)

$$
\operatorname{Res}_{V / V_{0}}\left(\operatorname{Princ}_{\Delta}(\phi)\right)=\operatorname{Princ}_{\Delta_{0}}\left(\operatorname{Res}_{V / V_{0}}(\phi)\right) .
$$

$$
\operatorname{Res}_{V / V_{0}}\left(\operatorname{Res}_{\Delta}(\phi)\right)=\operatorname{Res}_{\Delta_{0}}\left(\operatorname{Res}_{V / V_{0}}(\phi)\right) .
$$

Proof. Remark that

$$
N G_{\Delta} \subset \Delta_{0}^{-1} S(V)+\sum_{\nu \subset \Delta_{1}} N G_{\Delta_{0}} m_{\nu}
$$

Therefore, $\operatorname{Res}_{V / V_{0}}$ maps $N G_{\Delta}$ to $N G_{\Delta_{0}}$, and both members of equation (1) vanish on $N G_{\Delta}$.

Now consider an element $m_{\nu}=m_{\nu_{0}} m_{\nu_{1}}$ where $\nu$ is generating. If the length of $\nu_{1}$ is greater than 1 , both members of equation (1) vanish. If $\nu_{1}$ consists of one element, then $\nu_{0}$ generates $V_{0}$ and we obtain Assertion 1. Assertion 2 follows from the fact that $\operatorname{Res}_{V / V_{0}}$ is homogeneous of degree -1 . 


\section{Orlik-Solomon relations}

In this section, we describe the linear relations between the generators $\phi_{\sigma}$ $(\sigma \in \mathcal{B}(\Delta))$ of the space $S_{\Delta}$, and we construct bases of this space consisting of certain $\phi_{\sigma}$. Using iterated residues, we construct the dual bases as well.

For this, we begin by interpreting the space $S_{\Delta}$ in terms of the OrlikSolomon algebra associated to the hyperplane arrangement $\mathcal{H}^{*}(\Delta)$, see [6] Chapter 3. Recall that this algebra, which we denote by $A_{\Delta}$, is the subalgebra of rational differential forms on $V^{*}$ generated by the forms

$$
\omega_{\alpha}:=\frac{d \alpha}{\alpha}
$$

where $\alpha \in \Delta$. Clearly, $A_{\Delta}$ is graded by the degree of differential forms, and its top degree component is

$$
A_{\Delta}[r]=S_{\Delta} \otimes \wedge^{r} V
$$

It is known that the algebra $A_{\Delta}$ is the quotient of the free exterior algebra on symbols $e_{\alpha}(\alpha \in \Delta)$ by its ideal generated by the elements

$$
\sum_{j=1}^{s}(-1)^{j-1} e_{\alpha_{1}} \wedge \cdots \wedge \widehat{e_{\alpha_{j}}} \wedge \cdots \wedge e_{\alpha_{s}}
$$

where $\alpha_{1}, \ldots, \alpha_{s} \in \Delta$ are linearly dependent (see $[6] 3.5$ ). It follows that the space $A_{\Delta}[r]$ is generated by the elements

$$
\omega_{\left(\alpha_{1}, \ldots, \alpha_{r}\right)}:=\omega_{\alpha_{1}} \wedge \cdots \wedge \omega_{\alpha_{r}}=\frac{d \alpha_{1} \wedge \cdots \wedge d \alpha_{r}}{\alpha_{1} \cdots \alpha_{r}}
$$

where $\left(\alpha_{1}, \ldots, \alpha_{r}\right)$ is an ordered basis of $\Delta$. Moreover, the linear relations between the $\omega_{\left(\alpha_{1}, \ldots, \alpha_{r}\right)}$ are consequences of the relations

$$
\sum_{1 \leq j \leq r, c_{j} \neq 0}(-1)^{j-1} \omega_{\alpha_{1}, \ldots, \widehat{\alpha_{j}}, \ldots, \alpha_{r}, \alpha}=0
$$

where $\left(\alpha_{1}, \ldots, \alpha_{r}\right)$ is as above, and where $\alpha=\sum_{j=1}^{r} c_{j} \alpha_{j}$.

Finally, a basis of $A_{\Delta}[r]$ consisting of certain $\omega_{\left(\alpha_{1}, \ldots, \alpha_{r}\right)}$ can be defined as follows (see [2], [6] 3.2 and [7]). Choose an ordering $\left(\alpha_{1}, \alpha_{2}, \alpha_{3}, \ldots, \alpha_{N}\right)$ of $\Delta$. Consider the subset $B \subset \mathcal{B}(\Delta)$ consisting of the ordered bases $b=$ 
$\left(\alpha_{i_{1}}, \alpha_{i_{2}}, \ldots, \alpha_{i_{n}}\right)$ (listed with strictly increasing indices) such that for all $j \neq i_{p}$, the set $\left\{\alpha_{j}\right\} \cup\left\{\alpha_{i_{p}} ; i_{p}>j\right\}$ is linearly independent. Then the $\omega_{b}$ $(b \in B)$ are the desired basis.

Translating these results in terms of $S_{\Delta}$ leads to the following

Proposition 13 The set $\left(\phi_{b}\right)_{b \in B}$ is a basis of $S_{\Delta}$. Furthermore, the space of linear relations between the $\phi_{\sigma}(\sigma \in \mathcal{B}(\Delta))$ is generated by the "OrlikSolomon relations"

$$
r_{\sigma, \alpha}:=\phi_{\sigma}-\sum_{\beta \in \sigma} c_{\alpha \beta} \phi_{\sigma \cup\{\alpha\} \backslash\{\beta\}}
$$

where $\sigma \in \mathcal{B}(\Delta), \alpha \in \Delta \backslash \sigma$ and $\alpha=\sum_{\beta \in \sigma} c_{\alpha \beta} \beta$.

For completeness, we will present an a priori proof of this result; first, let us give an example.

Example. Let $V$ be a vector space with basis $e_{1}, e_{2}, e_{3}$. Consider the ordered set

$$
\Delta=\left(e_{1}, e_{2}, e_{3}, e_{1}+e_{2}, e_{2}+e_{3}, e_{1}+e_{2}+e_{3}\right)
$$

(the set of positive roots of a root system of type $A_{3}$ ). Then the set $B$ consists of

$$
b_{1}=\left(e_{1}, e_{2}, e_{3}\right), b_{2}=\left(e_{1}, e_{3}, e_{1}+e_{2}\right), b_{3}=\left(e_{1}, e_{2}, e_{2}+e_{3}\right),
$$

$b_{4}=\left(e_{1}, e_{1}+e_{2}, e_{2}+e_{3}\right), b_{5}=\left(e_{1}, e_{2}, e_{1}+e_{2}+e_{3}\right), b_{6}=\left(e_{1}, e_{3}, e_{1}+e_{2}+e_{3}\right)$.

Proof. Let $L$ be the free vector space with basis the elements $\phi_{\sigma}, \sigma \in \mathcal{B}(\Delta)$. Let $L R$ be the kernel of the natural map from $L$ to $R_{\Delta}$. By definition, $L R$ is the space of linear relations between the $\phi_{\sigma}$. We denote by $C$ the subspace of $L$ with basis $\left(\phi_{b}\right)_{b \in B}$, and by $O S R$ the subspace of $L R$ generated by the elements $r_{\sigma, \alpha}$. Let us show that $L=C+O S R$. If $\sigma=\left(\alpha_{i_{1}}, \alpha_{i_{2}}, \ldots, \alpha_{i_{r}}\right)$ is an ordered basis of $\Delta$, we set $|\sigma|=i_{1}+i_{2}+\cdots+i_{r}$. If $\sigma$ is not in $B$, then there exists a $j$ such that the set $\left\{\alpha_{j}\right\} \cup\left\{\alpha_{i_{p}} ; i_{p}>j\right\}$ is linearly dependent. Using the relation $r_{\sigma, \alpha_{j}}$, we replace $\phi_{\sigma}$ by a linear combination of elements $\phi_{\tau}$ where $\tau$ is obtained from $\sigma$ by replacing one of the elements $\alpha_{i_{p}}$ with $i_{p}>j$ by $\alpha_{j}$. It follows that the numbers $|\tau|$ are strictly smaller than $|\sigma|$, so that by induction, we obtain $L=C+O S R$. This shows that the set $\left(\phi_{b}\right)_{b \in B}$ generates $S_{\Delta}$, and that $L R$ is spanned by $C \cap L R$ and by $O S R$.

We now show that $C \cap L R=0$. We need to check that if $\sum_{b \in B} c_{b} \phi_{b}=0$ as a rational function, then all $c_{b}$ are equal to 0 . We prove this by induction on 
the number of elements in $\Delta$. Remark that all elements of $B$ contain $\alpha_{1}$. For a set $\kappa$ of $r-1$ linearly independent vectors, let $H(\kappa) \subset V$ be the hyperplane generated by $\kappa$. For a hyperplane $H \subset V$, set $\mathcal{B}(H):=\{\kappa \subset \Delta ; H(\kappa)=H\}$. We write

$$
\sum_{b \in B} c_{b} \phi_{b}=\alpha_{1}^{-1} \sum_{H} \phi_{H}
$$

with

$$
\phi_{H}:=\sum_{b, H\left(b \backslash\left\{\alpha_{1}\right\}\right)=H} \frac{c_{b}}{\prod_{\beta \in b \backslash\left\{\alpha_{1}\right\}} \beta} .
$$

Choose a hyperplane $H_{0}$ generated by $b \backslash\left\{\alpha_{1}\right\}$, for some $b \in B$. Then the residue operator $\operatorname{Res}_{V / H_{0}}$ kills all elements $\alpha_{1}^{-1} \phi_{H}$ except $\alpha_{1}^{-1} \phi_{H_{0}}$, which is mapped to $\phi_{H_{0}}$. Thus, $\phi_{H_{0}}=0$. But remark that if we consider the ordered set $\Delta_{0}=\Delta \cap H_{0}$, the set $B_{0}=B\left(\Delta_{0}\right)$ consists exactly of the elements $b_{0}$ such that $\left\{\alpha_{1}\right\} \cup b_{0} \in B$. We conclude by applying the induction hypothesis to the vector space $H_{0}$ and the system $\Delta \cap H_{0}$, for all $H_{0}$.

We see that giving an ordering of $\Delta$, the set $B$ is characterized as the unique generating family $\left(\phi_{b}\right)$ with $\sum_{b \in B}|b|$ minimum.

Following [7], we now construct the dual basis $\left(\phi^{b}\right)_{b \in B}$ of the basis $\left(\phi_{b}\right)$, by using iterated residues. In our framework, they can be introduced as follows.

Let $\alpha \in \Delta$ and let $\Delta \backslash k \alpha$ be the complement in $\Delta$ of the set of scalar multiples of $\alpha$. Denote by $\Delta / \alpha$ the image of $\Delta \backslash k \alpha$ in the quotient space $V / k \alpha$. Any $\phi \in S_{\Delta}$ has at worst a simple pole along $\alpha=0$. Thus, restriction of $\alpha \phi$ to $(\alpha=0)=(V / k \alpha)^{*}$ is a well defined element of $(\Delta / \alpha)^{-1} S(V / k \alpha)$; we denote it by $\operatorname{Res}_{\alpha}(\phi)$. This defines a linear map

$$
\operatorname{Res}_{\alpha}: S_{\Delta}=G_{\Delta}[-r] \rightarrow G_{\Delta / \alpha}[-r+1]=S_{\Delta / \alpha} .
$$

Given an ordered basis $\left(\beta_{1}, \ldots, \beta_{r}\right)$ of $\Delta$, we can iterate this construction to obtain a linear form

$$
\operatorname{Res}_{\beta_{1}} \operatorname{Res}_{\beta_{2}} \cdots \operatorname{Res}_{\beta_{r}}
$$

on $S_{\Delta}$. On the other hand, we have a complete flag

$$
0=V_{0} \subset V_{1} \subset \cdots \subset V_{r}=V
$$

(where each $V_{j}$ is spanned by $\beta_{r-j+1}, \beta_{r-j+2}, \ldots, \beta_{r}$ ) together with a non-zero element of each $\wedge^{j} V_{j}$. Thus, we obtain another linear form

$$
\operatorname{Res}_{V_{1} / V_{0}} \cdots \operatorname{Res}_{V_{r-1} / V_{r-2}} \operatorname{Res}_{V / V_{r-1}}
$$

on $S_{\Delta}$ identified with $S_{\Delta} \otimes \wedge^{r} V$. 
Proposition 14 1) (Orlik-Solomon-Terao) For any $\alpha \in \Delta$, the map Res $s_{\alpha}$ defines an exact sequence

$$
0 \rightarrow S_{\Delta \backslash k \alpha} \rightarrow S_{\Delta} \rightarrow S_{\Delta / \alpha} \rightarrow 0
$$

2) For any ordered basis $b=\left(\beta_{1}, \ldots, \beta_{r}\right)$ of $\Delta$, we have

$$
\operatorname{Res}_{\beta_{1}} \operatorname{Res}_{\beta_{2}} \cdots \operatorname{Res}_{\beta_{r}}=\operatorname{Res}_{V_{1} / V_{0}} \cdots \operatorname{Res}_{V_{r-1} / V_{r-2}} \operatorname{Res}_{V / V_{r-1}}
$$

with notation as above.

3) (Szenes) The dual basis of $\left(\phi_{b}\right)_{b \in B}$ is given by

$$
\phi^{b}=\operatorname{Res}_{\beta_{1}} \operatorname{Res}_{\beta_{2}} \cdots \operatorname{Res}_{\beta_{r}} .
$$

Proof. 1) is a consequence of [6] Theorem 3.126; a direct proof is a follows. Let $\sigma$ be a basis of $\Delta$ containing $\alpha$, and let $\sigma / \alpha$ be its image in $V / k \alpha$. Then $\sigma / \alpha$ is a basis of $\Delta / \alpha$ and all bases of $\Delta / \alpha$ are obtained in this way. Moreover, $\operatorname{Res}_{\alpha}\left(\phi_{\sigma}\right)$ is a non-zero multiple of $\phi_{\sigma / \alpha}$. It follows that $\operatorname{Res}_{\alpha}$ is surjective.

Clearly, the kernel of $\operatorname{Res}_{\alpha}$ contains $S_{\Delta \backslash k \alpha}$. Conversely, if $\phi \in S_{\Delta}$ is mapped to 0 by $\operatorname{Res}_{\alpha}$, then $\phi$ is defined on $(\alpha=0)$ and thus, $\phi \in R_{\Delta \backslash k \alpha}$. We can write $\phi=\phi_{1}+\phi_{2}$ where $\phi_{1} \in S_{\Delta \backslash k \alpha}$ and $\phi_{2} \in V^{*} R_{\Delta \backslash k \alpha}$. Then $\phi_{2}=\phi-\phi_{1}$ is in $S_{\Delta}$, too, whence $\phi_{2}=0$ and $\phi \in S_{\Delta \backslash k \alpha}$.

2) Let $b^{\prime}=\left(\beta_{1}^{\prime}, \ldots, \beta_{r}^{\prime}\right)$ be another ordered basis of $\Delta$. Consider the element

$$
\rho:=\operatorname{Res}_{\beta_{1}} \cdots \operatorname{Res}_{\beta_{r}}\left(\phi_{b^{\prime}}\right) .
$$

If $\operatorname{Res}_{\beta_{r}}\left(\phi_{b^{\prime}}\right)$ is non-zero, then we must have $\beta_{r}=t_{r} \beta_{\pi(r)}^{\prime}$ for some non-zero $t_{r} \in k$ and some index $\pi(r)$. If moreover $\operatorname{Res}_{\beta_{r-1}} \operatorname{Res}_{\beta_{r}}\left(\phi_{b^{\prime}}\right)$ is non-zero, then we must have $\beta_{r-1} \in t_{r-1} \beta_{\pi(r-1)}^{\prime}+k \beta_{\pi(r)}^{\prime}$ for some non-zero $t_{r-1} \in k$ and some $\pi(r-1) \neq \pi(r)$ (because $\beta_{r-1}$ is not a multiple of $\left.\beta_{r}\right)$. Continuing in this way, we see that either $\rho=0$ or there exists a permutation $\pi$ of $\{1, \ldots, r\}$ and non-zero $t_{1}, \ldots, t_{r} \in k$ such that

$$
\beta_{i} \in t_{i} \beta_{\pi(i)}^{\prime}+\sum_{j=i+1}^{r} k \beta_{\pi(j)}^{\prime}
$$

for all $i$. Then we have $\rho=t_{1} \cdots t_{r}$.

On the other hand, set

$$
\rho^{\prime}:=\operatorname{Res}_{V_{1} / V_{0}} \cdots \operatorname{Res}_{V / V_{r-1}}\left(\phi_{b^{\prime}}\right) .
$$


If $\operatorname{Res}_{V / V_{r-1}}\left(\phi_{b^{\prime}}\right) \neq 0$, then there exist a unique index $\pi^{\prime}(1)$ and a unique nonzero $t_{1}^{\prime} \in k$ such that $\beta_{\pi^{\prime}(1)}^{\prime} \in t_{1}^{\prime} \beta_{1}+\sum_{j=2}^{r} k \beta_{j}$. Further, $\beta_{i}^{\prime} \in \sum_{j=2}^{r} k \beta_{j}$ for all $i \neq \pi^{\prime}(1)$. If moreover $\operatorname{Res}_{V_{r-1} / V_{r-2}} \operatorname{Res}_{V / V_{r-1}}\left(\phi_{b^{\prime}}\right) \neq 0$, then $\beta_{\pi^{\prime}(r-1)}^{\prime} \in t_{2}^{\prime} \beta_{2}+$ $\sum_{j=3}^{r} k \beta_{j}$ for uniquely defined $\pi^{\prime}(r-1)$ and $t_{r-1}^{\prime}$. Further, $\beta_{i}^{\prime} \in \sum_{j=3}^{r} k \beta_{j}$ for all $i \notin\left\{\pi^{\prime}(1), \pi^{\prime}(2)\right\}$. Continuing, we obtain if $\rho^{\prime} \neq 0$ :

$$
\beta_{\pi^{\prime}(i)}^{\prime} \in t_{i}^{\prime} \beta_{i}+\sum_{j=i+1}^{r} k \beta_{i}
$$

for a permutation $\pi^{\prime}$ and non-zero $t_{1}^{\prime}, \ldots, t_{r}^{\prime}$; then we have $\rho^{\prime}=1 / t_{1}^{\prime} \cdots t_{r}^{\prime}$. This is equivalent to the set of conditions of the first part of the proof, with $\pi^{\prime}=\pi$ and $t_{i}^{\prime}=1 / t_{i}$.

3) Let $b^{\prime}=\left(\beta_{1}^{\prime}, \ldots, \beta_{r}^{\prime}\right) \in B$ such that $\operatorname{Res}_{\beta_{1}} \cdots \operatorname{Res}_{\beta_{r}}\left(\phi_{b^{\prime}}\right) \neq 0$. Let $\pi$ and $t_{1}, \ldots, t_{r}$ be as above; then $\beta_{r-1}, \beta_{\pi(r-1)}^{\prime}$ and $\beta_{r}=t_{r} \beta_{\pi(r)}^{\prime}$ are linearly dependent. Write $\beta_{r-1}=\alpha_{i}, \beta_{\pi(r-1)}^{\prime}=\alpha_{i^{\prime}}$ and $\beta_{r}=\alpha_{j}$, then $j>i$ and $j>i^{\prime}$. If $i>i^{\prime}$ then $\alpha_{i^{\prime}}, \beta_{r-1}$ and $\beta_{r}$ are linearly dependent, which contradicts the hypothesis $b \in B$. Similarly, we cannot have $i<i^{\prime}$. Thus, $i=i^{\prime}$, that is, $\beta_{r-1}=\beta_{\pi(r-1)}^{\prime}$. In this way we obtain $\beta_{q}=\beta_{\pi(q)}^{\prime}$ for all $q$; because $b$ and $b^{\prime}$ are in $B$, it follows that $b=b^{\prime}$.

\section{Laplace transform and Jeffrey-Kirwan residue}

Starting from now, we assume that $k=\mathbb{R}$. The Laplace transform associates to a polynomial function supported on an acute cone in $V$ a rational function on $V^{*}$. We will define the inverse Laplace transform, formally denoted by $\int_{V^{*}}^{\delta} e^{\langle y, h\rangle}[[\phi(y)]] d y$, of a meromorphic function $\phi$ on $V^{*}$ with poles on a set of hyperplanes. It depends of a choice of a chamber $\delta$ in $V^{*}$ and is a locally polynomial function on the dual cone $\delta^{\vee}$ of $\delta$.

Let $V$ be an oriented real vector space of dimension $r$. We denote by $o$ its orientation. We consider as before a finite subset $\Delta$ of $V \backslash\{0\}$, which spans $V$; we assume moreover that $-\alpha \in \Delta$ for all $\alpha \in \Delta$. A wall of $V$ is defined to be an hyperplane generated by $r-1$ linearly independent elements of $\Delta$. We denote by $\mathcal{H}(\Delta)$ the union of walls. This is a set of hyperplanes in $V$. The set

$$
V_{\text {reg, }, \Delta}:=V-\mathcal{H}(\Delta)
$$

is the set of regular elements in $V$. 
We define the vector space $\mathcal{P}_{\Delta}$ of locally polynomial functions on $V_{\text {reg, } \Delta}$. Elements of $\mathcal{P}_{\Delta}$ are given by polynomial functions on each connected component of $V_{\text {reg, } \Delta}$. The space $S\left(V^{*}\right)$ identifies to the space of polynomial functions on $V$. Thus the space $\mathcal{P}_{\Delta}$ is a module under the action of $S\left(V^{*}\right)$ by multiplication.

Let $C \subset V$ be an acute convex cone with non empty interior. Let $C^{\vee} \subset V^{*}$ be its (closed) dual cone. Then the interior of $C^{\vee}$ is not empty. We denote by $[C]$ the characteristic function of $C$, that is, the function with value 1 on $C$ and 0 outside $C$.

Let $f \in S\left(V^{*}\right)$ be a polynomial function on $V$. Let $d h$ be an element of $\wedge^{r} V^{*}$. As $V$ is oriented, we can integrate over $V$ a differential form $\alpha=$ $\phi(h) d h$ of maximal degree (here $\phi$ is an integrable function on $V$ ). We denote the integral over $V$ of such a differential form $\alpha$ by $\int_{V, o} \alpha$. A change of orientation produces a change of sign.

For each $y$ in the interior of $C^{\vee}$, the integral

$$
L^{o}(f[C])(y):=\int_{V, o} e^{-\langle y, h\rangle} f(h)[C](h) d h
$$

converges, and defines the Laplace transform of $f[C]$. If $C$ is generated by multiples of elements of $\Delta$, it is easy to see that $L^{o}(f[C])$ is given by the restriction to the interior of $C^{\vee}$ of a rational function on $V^{*}$ belonging to the subspace $G_{\Delta}$ of $R_{\Delta}$. We still denote this rational function by $L^{o}(f[C])(y)$. More exactly, as $L^{o}(f[C])(y)$ depends linearly on $d h$, we see that $L^{o}(f[C])(y)$ is a rational function with values in $\left(\wedge^{r} V^{*}\right)^{*}=\wedge^{r} V$. Furthermore it is clear that the map $L^{o}$ interchanges the action of $S\left(V^{*}\right)$ by multiplication on $\mathcal{P}_{\Delta}$ with its action by derivation on $G_{\Delta}$, up to the automorphism $P(h) \mapsto P(-h)$.

Let $\delta$ be a connected component of the set $V^{*}-\mathcal{H}^{*}(\Delta)$. Then $\delta$ is an open acute polyhedral cone in $V^{*}$, and $\delta^{\vee}$ is a closed acute polyhedral cone in $V$. We denote by $\mathcal{P}_{\Delta}(\delta)$ the subspace of $\mathcal{P}_{\Delta}$ spanned by functions $f(h)[C(\sigma)](h)$ where $f \in S\left(V^{*}\right)$ and $\sigma$ is a basis of $\Delta$ such that $C(\sigma) \subset \delta^{\vee}$; here $C(\sigma)$ denotes the closed convex cone generated by $\sigma$.

If $d h$ is a positive element of $\wedge^{\max } V^{*}$, we denote by $\operatorname{vol}(\sigma, d h)$ the volume of the parallelelepiped constructed on the basis $\sigma$ for the positive density $d h$ corresponding to the differential form $d h$. Specifically, if $d h=e^{1} \wedge e^{2} \wedge \cdots \wedge e^{r}$ and if $\sigma=\left\{\alpha_{1}, \ldots, \alpha_{r}\right\}$, we have $\operatorname{vol}(\sigma, d h)=\left|\operatorname{det}\left\langle\alpha_{i}, e^{j}\right\rangle_{i, j}\right|$. Finally, we denote by $L_{\delta}^{o}$ the restriction to $\mathcal{P}_{\Delta}(\delta)$ of the Laplace transform $L^{o}$. 
Theorem 15 Given any chamber $\delta$ in $V^{*}$, the Laplace transform

$$
L_{\delta}^{o}: \mathcal{P}_{\Delta}(\delta) \rightarrow G_{\Delta} \otimes \wedge^{\max } V
$$

is an isomorphism and commutes with the actions of $S\left(V^{*}\right)$ up to the automorphism $P(h) \mapsto P(-h)$.

We have, for $d h \in \wedge^{\text {max }} V^{*}$ positive (with respect to our choice of orientation o), and $\sigma$ a basis of $\Delta$ such that $C(\sigma) \subset \delta^{\vee}$,

$$
<L_{\delta}^{o}[C(\sigma)], d h>=\operatorname{vol}(\sigma, d h) \phi_{\sigma}
$$

that is, for $y \in \delta$, we have the equality of functions

$$
\int_{C(\sigma), o} e^{-\langle y, h\rangle} d h=\operatorname{vol}(\sigma, d h) \phi_{\sigma}(y) .
$$

Proof. The formula for the Laplace transform of $[C(\sigma)]$ is straightforward. It implies surjectivity of $L_{\delta}^{o}$ because this map is $S\left(V^{*}\right)$-linear, and the $S\left(V^{*}\right)$ module $G_{\Delta}$ is generated by the $\phi_{\sigma}$ where $\sigma$ is a basis of $\Delta$ such that $C(\sigma)$ is contained in $\delta^{\vee}$ (here we use the assumption that $\Delta$ is centrally symmetric).

For injectivity of $L_{\delta}^{o}$, we observe that any function $\phi \in \mathcal{P}_{\Delta}(\delta)$ is supported in the acute cone $\delta^{\vee}$, and that $\phi=0$ if and only if $\phi$ vanishes outside a set of measure zero. Moreover, the set of functions $h \mapsto e^{-\langle y, h\rangle}$ (where $y \in \delta$ ) is dense in the space of smooth, rapidly decreasing functions on $\delta^{\vee}$.

Consider the inverse $\left(L_{\delta}^{o}\right)^{-1}: G_{\Delta} \otimes \wedge^{\max } V \mapsto \mathcal{P}_{\Delta}(\delta)$. Via the projection map $\operatorname{Princ}_{\Delta}$, we can extend the map $\left(L_{\delta}^{o}\right)^{-1}$ to $\Delta^{-1} \hat{S}(V) \otimes \wedge^{\max } V$. Thus we set

$$
F_{\delta}^{o}(\phi \otimes d y):=\left(L_{\delta}^{o}\right)^{-1}\left(\operatorname{Princ}_{\Delta}(\phi) \otimes d y\right) .
$$

Thus, $F_{\delta}^{o}$ associates to any meromorphic function $\phi$ on $V^{*}$ with poles on the hyperplanes $\alpha=0$ a locally polynomial function on $\delta^{\vee}$. We denote $F_{\delta}^{o}(\phi \otimes d y)$ by the formal notation:

$$
\left(F_{\delta}^{o}(\phi \otimes d y)\right)(h)=\int_{V^{*}, o}^{\delta} e^{\langle y, h\rangle}[[\phi(y)]] d y .
$$

We now show that $F_{\delta}^{o}$ commutes with the actions of $S(V)$ by derivations on $\mathcal{P}_{\Delta}(\delta)$, and by multiplication on $R_{\Delta}$. 
Lemma 16 For any $\psi \in S(V)$ and $\phi \in G_{\Delta}$, we have

$$
\psi(\partial) F_{\delta}^{o}(\phi \otimes d y)=F_{\delta}^{o}(\psi \phi \otimes d y)
$$

Proof. It is enough to check this for $\psi=v \in V$. Then, for any $y \in \delta$, we have

$$
\begin{gathered}
\int_{V}\left(\partial(v) F_{\delta}^{o}(\phi)\right)(h) e^{-\langle y, h\rangle} d h-\langle v, y\rangle \phi(y) \\
=\int_{V}\left(\partial(v) F_{\delta}^{o}(\phi)\right)(h) e^{-\langle y, h\rangle} d h+\int_{V} F_{\delta}^{o}(\phi)(h) \partial(v)\left(e^{-\langle y, h\rangle}\right) d h \\
=\int_{V} \partial(v)\left(F_{\delta}^{o}(\phi)(h) e^{-\langle y, h\rangle}\right) d h=\int_{\Sigma} F_{\delta}^{o}(\phi)(h) e^{-\langle y, h\rangle} i_{v}(d h)
\end{gathered}
$$

where $\Sigma$ denotes the boundary of the support of $F_{\delta}^{o}(\phi)$; here the latter equality follows from Stokes' theorem. Because $\Sigma$ is a union of polyhedral cones of smaller dimensions, the function

$$
y \mapsto \int_{\Sigma} F_{\delta}^{o}(\phi)(h) e^{-\langle y, h\rangle} i_{v}(d h)
$$

is in $N G_{\Delta}$. We thus have

$$
L_{\delta}^{o}\left(\partial(v) F_{\delta}^{o}(\phi \otimes d y)\right)-v \phi \in N G_{\Delta}
$$

which implies our formula.

If $\phi \in S_{\Delta}$, the image $F_{\delta}^{o}(\phi \otimes d y)$ is a locally constant function on $V_{\text {reg, }, \Delta}$. Thus we obtain a number of residue maps defined by chambers $\gamma$ in $V$ and $\delta$ in $V^{*}$ :

$$
\begin{gathered}
R e s_{\gamma, \delta}: S_{\Delta} \otimes \wedge^{\max } V \rightarrow \mathbf{R}, \\
\left.\phi \otimes d y \mapsto\left(F_{\delta}^{o} \phi\right)\right|_{\gamma} .
\end{gathered}
$$

The formula of Theorem 15 determines $\operatorname{Res}_{\gamma, \delta}\left(\phi_{\sigma} \otimes d y\right)$ for $C(\sigma) \subset \delta^{\vee}$ and $d y$ a positive element of $\wedge^{r} V$. More precisely, if $d h$ is the dual measure to $d y$,

$$
\begin{gathered}
\operatorname{Res}_{\gamma, \delta}\left(\phi_{\sigma} \otimes d y\right)=\frac{1}{\operatorname{vol}(\sigma, d h)}, \quad \text { if } \gamma \subset C(\sigma), \\
\operatorname{Res}_{\gamma, \delta}\left(\phi_{\sigma} \otimes d y\right)=0, \quad \text { if } \gamma \cap C(\sigma)=\emptyset .
\end{gathered}
$$


As $F_{\delta}^{o}$ commutes with the action of differential operators with constant coefficients, we have

$$
F_{\delta}^{o}\left(P(\partial) \phi_{\sigma} \otimes d y\right)=P(-h) F_{\delta}^{o}\left(\phi_{\sigma} \otimes d y\right)(h)
$$

so that if $C(\sigma) \subset \delta^{\vee}$

$$
F_{\delta}^{o}\left(P(\partial) \phi_{\sigma} \otimes d y\right)(h)=\frac{1}{\operatorname{vol}(\sigma, d h)} P(-h)[C(\sigma)](h) .
$$

Proposition 17 (Jeffrey-Kirwan) For $\phi \in \hat{R}_{\Delta}$ and $h \in V$, we have

$$
F_{\delta}^{o}(\phi \otimes d y)(h)=F_{\delta}^{o}\left(\operatorname{Res}_{\Delta}\left(e^{h} \phi\right) \otimes d y\right) .
$$

Proof. It is sufficient to prove this formula for $\phi=P(\partial) \phi_{\sigma}$. As we have for $y \in V^{*}$ :

$$
\operatorname{Res}_{\Delta}\left(e^{h} \partial(y) \phi\right)=-\operatorname{Res}_{\Delta}\left(\left(\partial(y) e^{h}\right) \phi\right)=-\langle y, h\rangle \operatorname{Res}_{\Delta}\left(e^{h} \phi\right),
$$

we obtain

$$
\operatorname{Res}_{\Delta}\left(e^{h} P(\partial) \phi_{\sigma}\right)=P(-h) \operatorname{Res}_{\Delta}\left(e^{h} \phi_{\sigma}\right)=P(-h) \phi_{\sigma} .
$$

So we see, from Formula (11) above, that the equation of Proposition 17 is satisfied.

Proposition 17 provides an effective tool to compute the inverse Laplace transform of a rational function $\phi$ with poles on hyperplanes. Indeed, the function $\operatorname{Res}_{\Delta}\left(e^{h} \phi\right)$ is an element of $S_{\Delta}$ (depending of $h$ ), so that it can be written as a linear combination

$$
\operatorname{Res}_{\Delta}\left(e^{h} \phi\right)=\sum_{\sigma} c_{\sigma}(h) \phi_{\sigma} .
$$

The choice of a chamber $\delta$ determines a sign $\epsilon(\sigma, \delta)$ for which

$$
\phi_{\sigma}=\epsilon(\sigma, \delta) \phi_{\sigma^{\delta}}
$$

where the cone $\sigma^{\delta}$ has the same axes as $C(\sigma)$ and is contained in $\delta^{\vee}$. Thus, the restriction to a chamber $\gamma$ in $V$ of the inverse Laplace transform $F_{\delta}^{o}(\phi \otimes d y)$ 
is obtained by summing the polynomial terms $\epsilon(\sigma, \delta) c_{\sigma}(h) \operatorname{vol}(\sigma, d h)^{-1}$ for all $\sigma$ such that $\gamma \subset C\left(\sigma^{\delta}\right)$ :

$$
\left.F_{\delta}^{o}(\phi \otimes d y)\right|_{\gamma}=\sum_{\sigma, \gamma \subset C\left(\sigma^{\delta}\right)} \epsilon(\sigma, \delta) c_{\sigma}(h) \operatorname{vol}(\sigma, d h)^{-1} .
$$

This is Jeffrey-Kirwan algebraic formula.

\section{Example}

Let us consider a two-dimensional vector space $V$ with basis $\left(e_{1}, e_{2}\right)$. Let $\Delta=\left\{e_{1}, e_{2}, e_{1}+e_{2}\right\}$. Consider

$$
\phi\left(z_{1}, z_{2}\right)=\frac{1}{z_{1} z_{2}\left(z_{1}+z_{2}\right)}
$$

We have

$$
\begin{aligned}
\operatorname{Res}_{\Delta}\left(e^{h_{1} z_{1}+h_{2} z_{2}} \phi\left(z_{1}, z_{2}\right) \otimes d z_{1} d z_{2}\right)=\frac{h_{1} z_{1}+h_{2} z_{2}}{z_{1} z_{2}\left(z_{1}+z_{2}\right)} & \\
= & =\frac{h_{1}}{z_{2}\left(z_{1}+z_{2}\right)}+\frac{h_{2}}{z_{1}\left(z_{1}+z_{2}\right)} .
\end{aligned}
$$

If $\delta$ is the component $e_{1}>0, e_{2}>0$ of $V^{*}$, we then obtain the following picture for the inverse Laplace transform of $\frac{1}{z_{1} z_{2}\left(z_{1}+z_{2}\right)}$.

In the next section, we determine the change of $F_{\delta}^{o} \phi$ when jumping over a wall.

\section{The jump formula}

We consider, as in Section 5 , a real oriented vector space $(V, o)$ with a system of hyperplanes defined by $\Delta \subset V-\{0\}$. Let $\delta$ be a chamber in $V^{*}$, and let $F_{\delta}^{o}$ be the inverse Laplace transform. In this section, we relate the jumps of $F_{\delta}^{o}(\phi) \otimes d y$ across walls, with the poles of the function $\phi$ along the wall.

Let $\left(V_{0}, o_{0}\right)$ be an oriented wall with its system $\Delta_{0}=\Delta \cap V_{0}$. The wall $V_{0}$ separates $V$ in two half-spaces. Choose an equation $z$ of $V_{0}$ such that $o=z \wedge o_{0}$, and define

$$
\begin{aligned}
& V_{+}=\{h \in V,\langle z, h\rangle>0\}, \\
& V_{-}=\{h \in V,\langle z, h\rangle<0\} .
\end{aligned}
$$


If $U$ is a component of $\left(V_{0}\right)_{r e g, \Delta_{0}}$ there exists unique components $U_{ \pm}$of $V_{\text {reg, } \Delta}$ contained in $V_{ \pm}$and such that $U \subset \overline{U_{ \pm}}$.

Let $f \in \mathcal{P}_{\Delta}$ be a locally polynomial function on $V_{\text {reg, } \Delta}$. Then the restriction of $f$ to $U_{+}$(resp. $U_{-}$) is given by a polynomial function $f^{+}$(resp. $f^{-}$). We define the locally polynomial function $\operatorname{Jump}_{o / o_{0}}(f) \in \mathcal{P}_{\Delta_{0}}$ by the formula

$$
\left.\operatorname{Jump}_{o / o_{0}}(f)\right|_{U}=\left.f^{+}\right|_{U}-\left.f^{-}\right|_{U}
$$

Theorem 18 Let $\left(V_{0}, o_{0}\right)$ be an oriented wall. Let $\delta$ be a chamber in $V^{*}$ and $\delta_{0}$ a chamber in $V_{0}^{*}$ such that $\delta_{0}^{\vee} \subset \delta^{\vee}$. Then, for any $\phi \in \hat{R}_{\Delta}$, we have the Jump formula:

$$
\operatorname{Jump}_{o / o_{0}}\left(F_{\delta}^{o}\left(\phi \otimes d y_{0}\right)\right)=F_{\delta_{0}}^{o_{0}}\left(\operatorname{Res}_{V / V_{0}}(\phi \otimes d y)\right) .
$$

Proof. It is sufficient to prove this formula for $\phi \in G_{\Delta}$. (On $N G_{\Delta}$, both sides are equal to 0 , because $\operatorname{Res}_{V / V_{0}}$ maps $N G_{\Delta}$ to $\left.N G_{\Delta_{0}}\right)$. Thus it is sufficient to prove this formula for a derivative $\phi=P(\partial) \phi_{\sigma}$ of an element $\phi_{\sigma}$, with $C(\sigma) \subset \delta^{\vee}$. Then

$$
F_{\delta}^{o}(\phi \otimes d y)(h)=P(-h)[C(\sigma)](h) .
$$

If $V_{0}$ is not a wall of $C(\sigma)$, then $F_{\delta}^{o}(\phi \otimes d y)$ has no jump along $V_{0}$. Thus the left-hand side of the equality in Theorem 18 is equal to 0 . The right-hand side is also 0 , as there are at least 2 vectors in $\sigma$ which are not in $\Delta_{0}$.

If $V_{0}$ is a wall of $C(\sigma)$, there exists $\beta \in \Delta$ such that $\sigma=\sigma_{0} \cup\{\beta\}$ where $\sigma_{0}$ is a basis of $V_{0}$. Write $V=V_{0} \oplus \mathbb{R} \beta$. Write an element $h \in V$ as $h=h_{0}+h_{1} \beta$ with $h_{0} \in V_{0}$ and $h_{1} \in \mathbb{R}$. Then the left-hand side is the function $P\left(-h_{0}\right)\left[C\left(\sigma_{0}\right)\right]\left(h_{0}\right)$. If $P$ is divisible by $h_{1}$, then $\operatorname{Res}_{V / V_{0}}\left(P(\partial) \phi_{\sigma} \otimes d y\right)=0$. Thus, both sides vanish. If $P$ only depends on $h_{0}$, then

$$
\operatorname{Res}_{V / V_{0}}\left(P(\partial) \phi_{\sigma} \otimes d y\right)=P(\partial) \phi_{\sigma_{0}} \otimes d y_{0}
$$

whence the right-hand side is $P\left(-h_{0}\right)\left[C\left(\sigma_{0}\right)\right]\left(h_{0}\right)$.

As an application of the Jump formula, let us relate the behaviour at infinity of a function $\phi \in G_{\Delta}$ to the order of differentiability of its inverse Laplace transform. For a positive integer $n$, we say that $\phi$ vanishes at order $n$ at infinity if the rational function $t \mapsto t^{n-1} \phi(y+t z)$ is 0 at $\infty$ for all regular $y \in V^{*}$ and for all $z \in V^{*}$. Equivalently, $\psi \phi \in G_{\Delta}$ for any $\psi \in S(V)$ of degree at most $n-1$ (indeed, recall that $G_{\Delta}$ is the space of functions that vanish at infinity). 
Corollary 19 For a function $\phi \in G_{\Delta}$ and a non-negative integer $k$, the following conditions are equivalent:

1) $F_{\delta}^{o}(\phi \otimes d y)$ extends to a function of class $C^{k}$ on $V$.

2) $\phi$ vanishes at order $k+2$ at infinity.

Further, for a wall $V_{0}$ with equation $z_{0}=0$ and for $\phi$ satisfying (1) or (2), the following conditions are equivalent:

1)' $F_{\delta}^{0}(\phi \otimes d y)$ extends to a function of class $C^{k+1}$ along $V_{0}$.

2)' For any regular $z \in V^{*}$, the rational function $t \mapsto \phi\left(z+t z_{0}\right)$ vanishes at order $k+3$ at infinity.

Proof. Observe that $F_{\delta}^{o}(\phi \otimes d y)$ extends to a continuous function on $V$ if and only if it has no jumps along walls. This amounts to $\operatorname{Res}_{V / V_{0}}(\phi \otimes d y)=0$ for any wall $V_{0}$ (because $R e s_{V / V_{0}}$ maps $G_{\Delta} \otimes \wedge^{r} V$ to $G_{\Delta_{0}} \otimes \wedge^{r-1} V_{0}$, and $F_{\delta_{0}}^{o_{0}}$ is injective on the latter). Equivalently,

$$
\operatorname{Res}_{t=\infty}\left(\phi\left(z+t z_{0}\right) d t\right)=0
$$

for all regular $z$ and for all $z_{0}$. Because $\phi$ vanishes at infinity, this means that $\phi$ vanishes at order 2 there. This proves the equivalence of (1) and (2) in the case where $k=0$.

The general case follows by induction on $k$. Indeed, recall that

$$
\partial(v) F_{\delta}^{o}(\phi \otimes d y)=F_{\delta}^{o}(v \phi \otimes d y)=F_{\delta}^{o}\left(\operatorname{Princ}_{\Delta}(v \phi) \otimes d y\right)
$$

for any $v \in V$. Thus, using the induction hypothesis for $k-1$, assertion (1) is equivalent to: $\phi$ and $\operatorname{Princ}_{\Delta}(v \phi)$ vanish at order $k+1$ at infinity. Then $v \phi \in G_{\Delta}$ (because $\phi$ vanishes at order 2 at infinity) and (1) is equivalent to: $v \phi$ vanishes at order $k+1$ at infinity.

The proof of equivalence of (1)' and (2)' is similar.

\section{Orlik-Solomon relations and stratified Fourier transform}

We still consider a real vector space $V$ with a finite subset $\Delta \subset V \backslash\{0\}$ such that $\Delta$ spans $V$ and $\Delta=-\Delta$. We fix a Lebesgue measure $d h$ on $V$ and a chamber $\delta \subset V^{*}$. Changing slightly notation, the inverse Laplace transform $F_{\delta}$ associates to any element of $G_{\Delta}$ a locally polynomial function on $V_{\text {reg, }, \Delta}$. In this section, we associate to any element of $G_{\Delta}$ a piecewise polynomial 
function defined on all of $V$. This assignement will depend on the choices of a chamber $\delta$ in $V^{*}$ and of a chamber $\gamma$ in $V$; it will be denoted by $F_{\gamma, \delta}$. The piecewise polynomial function $F_{\gamma, \delta}(\phi)$ will extend the locally polynomial function $F_{\delta}(\phi)$, and will be the continuous extension of $F_{\delta}(\phi)$ if it exists. We will use the function $F_{\gamma, \delta}(\phi)$ in part III of this article, in connection with the definition of Eisenstein series.

Denote by $\mathcal{P} \mathcal{P}_{\Delta}$ the vector space of functions on $V$ spanned by functions $P[C]$ where $P \in S\left(V^{*}\right)$ and $C$ is a closed polyhedral cone with axes in $\Delta$. Then $\mathcal{P} \mathcal{P}_{\Delta}$ is a $S\left(V^{*}\right)$-submodule of the module of piecewise polynomial functions on $V$ (for the stratification where the open strata are the chambers, and the closures of other strata are proper faces of closures of chambers). We begin by constructing a morphism of $S\left(V^{*}\right)$-modules from $G_{\Delta}$ to a quotient of $\mathcal{P} \mathcal{P}_{\Delta}$. This morphism will depend on the choice of a chamber $\gamma$ in $V$, will be denoted by $F_{\gamma}$, and will be called the formal Fourier transform.

For a basis $\sigma$ of $V$, we denote by $|\operatorname{det}(\sigma)|$ the volume of the parallelepiped constructed on $\sigma$. We set

$$
a_{\sigma}:=|\operatorname{det}(\sigma)| \phi_{\sigma}=\frac{|\operatorname{det}(\sigma)|}{\prod_{\alpha \in \sigma} \alpha},
$$

an element of $S_{\Delta}$. Remark that $a_{\sigma}$ does not change if we multiply elements in $\sigma$ by positive constants. The Orlik-Solomon relations are more naturally expressed in terms of the $a_{\sigma}$, as shown by the following result, an easy consequence of Theorem 1 and Proposition 13 .

Proposition 20 Let $\sigma$ be a basis of $\Delta$, let $\alpha \in \Delta \backslash \sigma$ and let $\alpha=\sum_{\beta \in \sigma} c_{\alpha \beta} \beta$ be the expansion of $\alpha$ in the basis $\sigma$. Then the elements $\left(a_{\sigma}\right)_{\sigma \in \mathcal{B}(\Delta)}$ verify the relations

$$
a_{\sigma}=\sum_{\beta \in \sigma, c_{\alpha \beta} \neq 0} \operatorname{sign}\left(c_{\alpha \beta}\right) a_{\sigma \cup\{\alpha\} \backslash\{\beta\}} .
$$

Furthermore, if $\mathcal{M}$ is a $S\left(V^{*}\right)$-module and $\left(A_{\sigma}\right)_{\sigma \in \mathcal{B}(\Delta)}$ is a family in $\mathcal{M}$ verifying the relations (OS), then there exists a unique map $A: R_{\Delta} \rightarrow \mathcal{M}$ such that

1) the map $A$ commutes with the action of $S\left(V^{*}\right)$.

2) For all $\sigma \in \mathcal{B}(\Delta)$, we have $A\left(a_{\sigma}\right)=A_{\sigma}$.

3) $A\left(N G_{\Delta}\right)=0$. 
Remark that the relations (OS) have coefficients equal to \pm 1 . It makes thus sense to find elements in an abelian group, satisfying these relations. The group $\mathcal{C}(V)$ generated by characteristic functions of locally closed polyhedral cones in $V$ will be very useful to construct such elements $A_{\sigma}$.

We introduce some notation. A polyhedral cone in $V$ is a closed convex cone $C \subset V$ (with vertex at 0 ) which is generated by finitely many vectors $h_{1}, \ldots, h_{n}$; we set $C=C\left(h_{1}, \ldots, h_{n}\right)$. For $A$ a subset of $V$, we denote by [A] the characteristic function of $A$, i.e., the function on $V$ with value 1 on $A$ and 0 outside $A$. We denote by $\mathcal{C}(V)$ the additive group of integral valued functions on $V$, generated by all characteristic functions of polyhedral cones.

For any closed convex cone $C$, we denote by $C^{0}$ the relative interior of $C$, i.e., the interior of $C$ in the affine space generated by $C$. Observe that $\mathcal{C}(V)$ contains the characteristic functions of relative interiors of polyhedral cones, and more generally, the characteristic functions of locally closed polyhedral cones. The subgroup of $\mathcal{C}(V)$ generated by characteristic functions of polyhedral cones which contain lines is denoted by $\mathcal{L C}(V)$. For example if $\alpha \in V$ is nonzero, then

$$
[C(-\alpha)]+\left[C(\alpha)^{0}\right] \in \mathcal{L C}(V) .
$$

We denote by $\mathcal{C}_{\Delta}$ the subspace of $\mathcal{C}(V)$ generated by characteristic functions of polyhedral cones $C(\kappa)$ where $\kappa \subset \Delta$. Then, by definition, $\mathcal{P} \mathcal{P}_{\Delta}$ is the $S\left(V^{*}\right)$-module generated by $\mathcal{C}_{\Delta}$. We denote by $\mathcal{L C}_{\Delta}$ the subspace of $\mathcal{C}_{\Delta}$ generated by functions $[C(\kappa)]$ where $\kappa \subset \Delta$ and $C(\kappa)$ contains a line.

Let $p \in V$ and let $C \subset V$ be a polyhedral cone with non-empty interior, such that $p$ lies in no hyperplane generated by a facet of $C$. Set

$$
C_{p}^{\prime}:=\left\{h \in C \mid \text { the segment }[h, p] \text { meets } C^{0}\right\} \text {. }
$$

Then $C_{p}^{\prime}$ is equal to $C$ minus the union of its facets which generate a hyperplane separating $C^{0}$ and $p$. In particular, $C_{p}^{\prime}$ is a locally closed polyhedral cone. If moreover $C=C(\sigma)$ where $\sigma \in \mathcal{B}(\Delta)$, and $p=\sum_{\alpha \in \sigma} p_{\alpha} \alpha$ is in $V_{\text {reg, }, \Delta}$, then we obtain easily

$$
C(\sigma)_{p}^{\prime}:=C\left(\alpha, p_{\alpha}>0\right)+C\left(\alpha, p_{\alpha}<0\right)^{0} .
$$

In particular, the cone $C(\sigma)_{p}^{\prime}$ depends only of the chamber $\gamma$ which contains $p$. Thus, we denote it by $C(\sigma)_{\gamma}^{\prime}$.

Define a map

$$
A_{\gamma}: \mathcal{B}(\Delta) \rightarrow \mathcal{C}_{\Delta}
$$


by

$$
A_{\gamma}(\sigma)=\left[C(\sigma)_{\gamma}^{\prime}\right]
$$

Theorem 21 For any chamber $\gamma$ in $V$, the family of elements $\left(A_{\gamma}(\sigma)\right)_{\sigma \in \mathcal{B}(\Delta)}$ verify the relations $(O S)$ in the quotient group $\mathcal{C}_{\Delta} / \mathcal{L C}_{\Delta}$.

Proof. Because of the relation $[C(-\alpha)]=-\left[C(\alpha)^{0}\right]$ modulo $\mathcal{L C}_{\Delta}$ we see that the image of the element $A_{p}(\sigma)$ in $\mathcal{C}_{\Delta} / \mathcal{L C}_{\Delta}$ changes sign, if we flip one of the elements $\beta_{j}$ in $\sigma=\left(\beta_{1}, \beta_{2}, \cdots, \beta_{r}\right)$ to $-\beta_{j}$. We thus may assume that the relation is

$$
\alpha=\beta_{1}+\beta_{2}+\cdots+\beta_{s}
$$

for some $s \leq r$. Then the cones $C\left(\sigma \cup\{\alpha\} \backslash\left\{\beta_{j}\right\}\right)(1 \leq j \leq s)$ are the maximal cones in a polyhedral subdivision of $C(\sigma)$, and we conclude by the lemma below.

Lemma 22 Let $C \subset V$ be a polyhedral cone. Let $C_{1}, \ldots, C_{n}$ be the maximal cones of a polyhedral subdivision of $C$. Let $p \in V$ such that $p$ lies in no hyperplane generated by a facet of some $C_{i}$. Then $C_{p}^{\prime}$ is the disjoint union of $C_{1, p}^{\prime}, \ldots, C_{n, p}^{\prime}$.

Proof. Clearly, each $C_{i, p}^{\prime}$ is contained in $C_{p}^{\prime}$. Conversely, let $x \in C_{p}^{\prime}$. If $x$ lies in no $C_{i, p}^{\prime}$ then the segment $[x, p] \cap C^{0}$ has a non-empty interior in $[x, p]$ and is contained in the union of all facets of the $C_{i}$. It follows that this segment is contained in a facet of some $C_{i}$. Thus, $p$ is in the hyperplane generated by this facet, a contradiction. So $x \in C_{i, p}^{\prime}$ for some $i$. Assume that $x \in C_{j, p}^{\prime}$ for some $j \neq i$. Then $[x, p] \cap C_{i}^{0}$ and $[x, p] \cap C_{j}^{0}$ are disjoint segments with non-empty interiors in $[x, p]$. Moreover, because $x \in C_{i} \cap C_{j}$, the closures of both segments contain $x$, a contradiction.

We denote by $\mathcal{L} \mathcal{P}_{\Delta}$ the $S\left(V^{*}\right)$-submodule of $\mathcal{P} \mathcal{P}_{\Delta}$ generated by $\mathcal{L C}_{\Delta}$, that is, the space of piecewise polynomial functions which are polynomial in at least one direction. By the preceeding theorem, together with Proposition 20, each choice of a chamber $\gamma$ in $V$ defines a morphism of $S\left(V^{*}\right)$-modules $F_{\gamma}$ from $G_{\Delta}$ to the quotient space $\mathcal{P} \mathcal{P}_{\Delta} / \mathcal{L} \mathcal{P}_{\Delta}$.

Definition 23 Let $\gamma$ be a connected component of $V_{\text {reg, } \Delta}$. We denote by

$$
F_{\gamma}: R_{\Delta} \rightarrow \mathcal{P} \mathcal{P}_{\Delta} / \mathcal{L} \mathcal{P}_{\Delta}
$$


the unique map such that

1) $F_{\gamma}$ commutes with the action of $S\left(V^{*}\right)$ up to the automorphism $P(h) \mapsto$ $P(-h)$.

2) $F_{\gamma}\left(|\operatorname{det}(\sigma)| \phi_{\sigma}\right)=\left[C(\sigma)_{\gamma}^{\prime}\right]$ for all $\sigma \in \mathcal{B}(\Delta)$.

3) $F_{\gamma}\left(N G_{\Delta}\right)=0$

We call $F_{\gamma}$ the formal Fourier transform.

Now we construct a lift of $F_{\gamma}: R_{\Delta} \rightarrow \mathcal{P} \mathcal{P}_{\Delta} / \mathcal{L} \mathcal{P}_{\Delta}$ to $\mathcal{P} \mathcal{P}_{\Delta}$. In other words, we associate to any element of $R_{\Delta}$ a piecewise polynomial function on $V$, compatibly with $F_{\gamma}$. We may do this by specifying a chamber in $V^{*}$, as shown by

Lemma 24 Let $\delta$ be a chamber in $V^{*}$ and let $\phi \in R_{\Delta}$. Then $F_{\gamma}(\phi)$ has a unique representative with support in $\delta^{\vee}$.

Proof. Let $\alpha \in \Delta$, then $\alpha$ or $-\alpha$ is in $\delta^{\vee}$. Using the relation $[C(-\alpha)]+$ $\left[C(\alpha)^{0}\right] \in \mathcal{L C}_{\Delta}$, we see that $C(\kappa)$ has a representative with support in $\delta^{\vee}$, for any linearly independent $\kappa \subset \Delta$. This shows existence. For uniqueness, it is enough to check that any $f \in \mathcal{L} \mathcal{P}_{\Delta}$ with support in some acute cone $C$ must be zero. This is shown in the proof of [1] Theorem 1.4 for $f \in \mathcal{L C}(V)$; this proof adapts with minor changes, as follows. Embed $\mathcal{L} \mathcal{P}_{\Delta}$ into the vector space $\mathcal{F}\left(V^{*}\right)$ of functions on $V^{*}$. The additive group of $V^{*}$ acts on $\mathcal{F}\left(V^{*}\right)$ by translations; we denote by $z \mapsto T(z)$ this action. For a polyhedral cone $C$ which contains a line $l$, we have $(1-T(z))[C]=0$ for all $z \in l$. Thus, for $P \in S\left(V^{*}\right)$, we have

$$
(1-T(z))^{N}(P[C])=0
$$

whenever $N>\operatorname{deg}(P)$. Because $f \in \mathcal{L P}_{\Delta}$, it follows that there exist $z_{1}, \ldots, z_{n} \in V^{*} \backslash\{0\}$ (non necessarily distinct) such that

$$
\prod_{j=1}^{n}\left(1-T\left(t_{j} z_{j}\right)\right) f=0
$$

for all $t_{j} \in \mathbb{R}$. Moreover, we can find $h \in V$ such that $h>0$ on $C \backslash\{0\}$ and that $\left\langle h, z_{j}\right\rangle \neq 0$ for all $j$. Replacing $z_{j}$ by $-z_{j}$, we may assume that $\left\langle h, z_{j}\right\rangle<0$ for all $j$. Let $w \in V^{*}$. We can choose $A>0$ such that

$$
\left\langle h, w+\sum_{j \in J} t_{j} z_{j}\right\rangle<0
$$


for any non-empty subset $J$ of $\{1, \ldots, n\}$ and for $t_{j}>A$. We have

$$
0=\left(\prod_{j=1}^{n}\left(1-T\left(-t_{j} z_{j}\right) f\right)(w)=\sum_{J \subset\{1, \ldots, n\}}(-1)^{|J|} f\left(w+\sum_{j \in J} t_{j} z_{j}\right)\right.
$$

By assumption, $f$ is identically zero on the open half-space $h<0$. It follows that $f(w)=0$.

We denote by $F_{\gamma, \delta}(\phi)$ the representative of $F_{\gamma}(\phi)$ with support in $\gamma^{\vee}$. Let us compute $F_{\gamma, \delta}\left(a_{\sigma}\right)$ for $\sigma \in \mathcal{B}(\Delta)$. Write

$$
a_{\sigma}=\epsilon(\sigma, \delta) a_{\sigma^{\delta}}
$$

where $\epsilon(\sigma, \delta)= \pm 1$ and the cone $C\left(\sigma^{\delta}\right)$ has the same axes as $C(\sigma)$ and is contained in $\delta^{\vee}$. Then, by definition

$$
F_{\gamma, \delta}\left(a_{\sigma}\right)=\epsilon(\sigma, \delta)\left[C\left(\sigma^{\delta}\right)\right]_{\gamma}^{\prime}
$$

These elements $F_{\gamma, \delta}\left(a_{\sigma}\right)$ satisfy the Orlik-Solomon relations in the space $\mathcal{P} \mathcal{P}_{\Delta}$.

Definition 25 Let $\gamma$ be a chamber in $V$ and let $\delta$ be a chamber in $V^{*}$. We denote by

$$
F_{\gamma, \delta}: R_{\Delta} \rightarrow \mathcal{P} \mathcal{P}_{\Delta}
$$

the unique map such that

1) $F_{\gamma, \delta}$ commutes with the action of $S\left(V^{*}\right)$ up to the automorphism $P(h) \mapsto$ $P(-h)$.

2) $F_{\gamma, \delta}\left(|\operatorname{det}(\sigma)| \phi_{\sigma}\right)=\left[C(\sigma)_{\gamma}^{\prime}\right]$ for all $\sigma \in \mathcal{B}(\Delta)$ such that $C(\sigma) \subset \delta^{\vee}$.

3) $F_{\gamma, \delta}\left(N G_{\Delta}\right)=0$.

We call $F_{\gamma, \delta}$ the stratified Fourier transform.

We now express $F_{\gamma, \delta}(\phi)$ in terms of $F_{\delta}(\phi)$.

Proposition 26 Let $\gamma$ be a chamber of $V$, let $p \in \gamma$ and let $\delta$ be a chamber in $V^{*}$. Then we have for any $\phi \in G_{\Delta}$ and $h \in V$ :

$$
F_{\gamma, \delta}(\phi)(h)=\lim _{\epsilon \rightarrow 0, \epsilon>0} F_{\delta}(\phi)(h+\epsilon p) .
$$

In particular, $F_{\gamma, \delta}(\phi)$ is an extension of $F_{\delta}(\phi)$ to the whole of $V$, and is the continuous extension if it exists. 
Proof. Observe first that the formula makes sense: because $p$ is regular, $h+\epsilon p$ is regular for $\epsilon$ sufficiently small and $\epsilon>0$. If the formula holds for $\phi$ then it holds for $P(\partial) \phi$ where $P \in S\left(V^{*}\right)$, because both $F_{\delta}$ and $F_{\gamma, \delta}$ are $S\left(V^{*}\right)$-linear. Thus it suffices to check the formula for $\phi=\phi_{\sigma}$ where $C(\sigma) \subset \gamma^{\vee}$. Then $F_{\gamma, \delta}(\phi)=\left[C(\sigma)_{p}^{\prime}\right]$ whereas $F_{\delta}(\phi)$ is restriction of $[C(\sigma)]$ to $V_{\text {reg, }, \Delta}$. But

$$
\left[C(\sigma)_{p}^{\prime}\right](h)=\lim _{\epsilon \rightarrow 0, \epsilon>0}[C(\sigma)](h+\epsilon p)
$$

as follows from the definition of $C_{p}^{\prime}$.

\section{References}

[1] M. Brion, M. Vergne: Residue formulae, vector partition functions and lattice points in rational polytopes, J. Amer. Math. Soc. 10 (1997), 797-833.

[2] I. M. Gelfand, A. Zelevinskii: Algebraic and combinatorial aspects of the general theory of hypergeometric functions, Functional Analysis Appl. 20 (1986), 183-197.

[3] V. Guillemin, J. Kalkman: The Jeffrey-Kirwan localization theorem and residue operations in equivariant cohomology, J. Reine Angew. Math. 470 (1996), 123-142.

[4] L. C. Jeffrey, F. C. Kirwan: Localisation for non abelian group actions, Topology 34 (1995), 291-327.

[5] L. C. Jeffrey, F. C. Kirwan: Localization and the quantization conjecture, Topology 36 (1997) 647-693.

[6] P. Orlik, H. Terao: Arrangements of Hyperplanes. Grundlehren der Mathematischen Wissenschaften 300, Springer-Verlag, Berlin, 1992.

[7] A. Szenes : Iterated residues and multiple Bernouilli polynomials, International Mathematical Research Notices, 18 (1998), 937-956. 